\title{
NON-HOPF REAL HYPERSURFACES WITH CONSTANT PRINCIPAL CURVATURES IN COMPLEX SPACE FORMS
}

\author{
JOSÉ CARLOS DÍAZ-RAMOS AND MIGUEL DOMÍNGUEZ-VÁZQUEZ
}

\begin{abstract}
We classify real hypersurfaces in complex space forms with constant principal curvatures and whose Hopf vector field has two nontrivial projections onto the principal curvature spaces.

In complex projective spaces such real hypersurfaces do not exist. In complex hyperbolic spaces these are holomorphically congruent to open parts of tubes around the ruled minimal submanifolds with totally real normal bundle introduced by Berndt and Brück. In particular, they are open parts of homogenous ones.
\end{abstract}

\section{INTRODUCTION}

A homogeneous submanifold of a Riemannian manifold is an orbit of the action of a closed subgroup of the isometry group of the ambient manifold. One of the aims of submanifold geometry is to classify homogeneous submanifolds of a given manifold and to characterize them in terms of geometric data. Of particular interest are homogeneous hypersurfaces, which arise as principal orbits of cohomogeneity one actions. Obviously, homogeneous hypersurfaces have constant principal curvatures, that is, the eigenvalues of their shape operator are constant. It is an outstanding problem to determine under which conditions hypersurfaces with constant principal curvatures are open parts of homogeneous ones.

In spaces of constant curvature, a hypersurface has constant principal curvatures if and only if it is isoparametric. The classification of isoparametric hypersurfaces was achieved by Segre [20] in Euclidean spaces and by Cartan [9] in real hyperbolic spaces. They all are open parts of homogeneous ones. The situation is more involved in spheres. Cartan classified hypersurfaces with $g \in\{1,2,3\}$ constant principal curvatures in spheres. Subsequently, Hsiang and Lawson [12] classified homogeneous hypersurfaces in spheres; they have $g \in\{1,2,3,4,6\}$ principal curvatures. Then, Münzner [18] showed that $g \in\{1,2,3,4,6\}$ for isoparametric hypersurfaces in general. Surprisingly, for $g=4$ there are isoparametric hypersurfaces that are not homogeneous [13]. Recently, Cecil, Chi and Jensen [10], and Immervoll [14] showed that, with a few possible exceptions, hypersurfaces with $g=4$ constant principal curvatures are among the known homogeneous and inhomogeneous examples. Some progress has been made for $g=6$ by Abresch [1] and Dorfmeister and Neher [11], but the problem remains open in full generality. See [24] for a survey.

2000 Mathematics Subject Classification. Primary 53C40, Secondary 53C55, 53C35.

Key words and phrases. Hopf hypersurfaces, homogeneous hypersurfaces, constant principal curvatures.

The first author has been supported by a Marie-Curie European Reintegration Grant (PERG04-GA2008-239162). The second author has been supported by the FPU programme of the Spanish Government. Both authors have been supported by project MTM2006-01432 (Spain). 
The problem is even more difficult in complex space forms. See [19] for a survey on this and related topics. By $c \neq 0$ we denote the constant holomorphic sectional curvature of a complex space form; thus, if $c>0$ (resp. $c<0$ ) we have a complex projective (resp. hyperbolic) space $\mathbb{C} P^{n}(c)$ (resp. $\left.\mathbb{C} H^{n}(c)\right)$. We denote by $J$ its Kähler structure. Let $M$ be a real hypersurface of a complex space form and $\xi$ a (local) unit normal vector field. Then, $J \xi$ is tangent to $M$ and is called the Hopf vector field of $M$. The hypersurface $M$ is said to be Hopf if $J \xi$ is a principal curvature vector field. The motivation for our work is to address the classification of real hypersurfaces with constant principal curvatures in complex space forms. We briefly summarize the current state of the problem.

Assume $M$ is a real hypersurface of a complex space form with $g$ distinct constant principal curvatures. For $p \in M$ denote by $h(p)$ the number of nontrivial projections of $J \xi_{p}$ onto the principal curvature spaces of $M$. Clearly, this function is integer-valued and $M$ is Hopf if and only if $h=1$. The classification of homogeneous real hypersurfaces in complex projective spaces $\mathbb{C} P^{n}(c)$ was derived by Takagi [21. It follows from this classification that $g \in\{2,3,5\}$. A remarkable feature of homogeneous real hypersurfaces in $\mathbb{C} P^{n}(c)$ is that they are Hopf. Subsequently, Takagi classified real hypersurfaces with $g \in\{2,3\}$ constant principal curvatures [22], [23] ([25] for $n=2, g=3$ ). It follows from his work that they all are Hopf and open parts of homogeneous ones. Kimura [15] classified Hopf real hypersurfaces with constant principal curvatures in $\mathbb{C} P^{n}$ and showed that these are open parts of homogeneous ones. No examples are known of real hypersurfaces with constant principal curvatures in $\mathbb{C} P^{n}(c)$ with $h>1$. Surprisingly, in $\mathbb{C} H^{n}(c)$ there are non-Hopf homogeneous real hypersurfaces. The first example was discovered by Lohnherr [16] and further examples were given by Berndt and Brück [3], [4]. We refer to \$2.2 for a brief introduction and to [7] for a deeper study of their geometry. Berndt and Tamaru obtained in [8] the classification of cohomogeneity one actions on $\mathbb{C} H^{n}(c)$. The number of principal curvatures of the homogeneous examples is $g \in\{2,3,4,5\}$. Montiel [17] classified real hypersurfaces with $g=2$ constant principal curvatures in $\mathbb{C} H^{n}(c)(n \geq 3)$. Berndt and the first author solved the case $g=3$ and $g=2, n=2$ [5], 6]. It follows from [17] that $h=1$ when $g=2$, and from [5] and [6] we get $h \leq 2$ if $g=3$. Hopf real hypersurfaces with constant principal curvatures in $\mathbb{C} H^{n}(c)$ were classified by Berndt [2] and they all are open parts of homogeneous ones. To our knowledge, 5] and [6] are the first classifications of this kind involving non-Hopf real hypersurfaces. Nothing is known about $h$ if $g \geq 4$.

Our aim in this paper is to carry out the next natural step after Berndt and Kimura's classification of Hopf real hypersurfaces with constant principal curvatures in $\mathbb{C} P^{n}(c)$ and $\mathbb{C} H^{n}(c)$. Thus, we classify real hypersurfaces with constant principal curvatures whose Hopf vector field $J \xi$ has $h=2$ nontrivial projections onto the principal curvature spaces.

Main Theorem. We have:

(a) There are no real hypersurfaces with constant principal curvatures in $\mathbb{C P}^{n}(c), n \geq 2$, whose Hopf vector field has $h=2$ nontrivial projections onto the principal curvature spaces.

(b) Let $M$ be a connected real hypersurface in $\mathbb{C} H^{n}(c), n \geq 2$, with constant principal curvatures and whose Hopf vector field has $h=2$ nontrivial projections onto the principal 
curvature spaces of $M$. Then, $M$ has $g \in\{3,4\}$ principal curvatures and is holomorphically congruent to an open part of:

(i) a ruled minimal real hypersurface $W^{2 n-1} \subset \mathbb{C} H^{n}(c)$ or one of the equidistant hypersurfaces to $W^{2 n-1}$, or

(ii) a tube around a ruled minimal Berndt-Brück submanifold $W^{2 n-k} \subset \mathbb{C} H^{n}(c)$ with totally real normal bundle, for some $k \in\{2, \ldots, n-1\}$.

In particular, $M$ is an open part of a homogeneous real hypersurface of $\mathbb{C} H^{n}(c)$.

The ruled minimal submanifolds $W^{2 n-k} \subset \mathbb{C} H^{n}(c)$ are homogeneous and have totally real normal bundle of rank $k \in\{1, \ldots, n-1\}$. Actually, $W^{2 n-1}$ was discovered by Lohnherr [16. Then, Berndt studied the geometry of the equidistant hypersurfaces to $W^{2 n-1} 3$. This construction was generalized by Berndt and Brück in [4]. Both $W^{2 n-1}$ and any of its equidistant hypersurfaces have $g=3$ principal curvatures. The tubes around $W^{2 n-k}$, $k \in\{2, \ldots, n-1\}$ have $g=4$ principal curvatures if $r \neq(1 / \sqrt{-c}) \log (2+\sqrt{3})$ and $g=3$ principal curvatures if $r=(1 / \sqrt{-c}) \log (2+\sqrt{3})$. See [7] for a detailed description.

The proof is as follows. First we use the Gauss and Codazzi equations to derive some algebraic properties of the eigenvalue structure of the shape operator. The methods used for this are similar to those of [5], although a bit more general. We would like to emphasize that whenever we use a method similar to one in [5] we explicitly point it out and skip the details as much as possible. On the other hand, we focus on the new techniques and results, especially on Subsection 3.4. The most crucial step of the proof is to show that the number $g$ of constant principal curvatures satisfies $g \leq 4$. For this we use a novel approach based on the study of some inequalities satisfied by the principal curvatures. Using standard Jacobi field theory one can deduce the geometry of the focal submanifolds of these hypersurfaces and then the result follows from a rigidity result in [7.

The paper is organized as follows. In Section 2 we introduce the basic elements of our paper. Subsection 2.1 is devoted to present the equations of submanifold geometry that we will use in the rest of the paper. In $\$ 2.2$ we briefly describe the ruled minimal Berndt-Brück submanifolds $W^{2 n-k}$. We prove our Main Theorem in Section 3. The proof is divided in several steps. Some vector fields and functions arise naturally in our proof ( $\$ 3.1$ and $\$ 3.21$ ). We get some of their properties in Subsection 3.3. In $\$ 3.4$ we show that the number $g$ of principal curvatures satisfies $g \in\{3,4\}$. We summarize all the eigenvalue structure in \$3.5. In Subsection 3.6 we use standard Jacobi field theory to finish the proof of the Main Theorem.

\section{Preliminaries}

In this section we introduce the basic notation of this paper. We write down the Gauss and Codazzi equations of a hypersurface in a complex space form and derive some basic consequences. Then, we briefly mention how the examples of the Main Theorem are constructed.

2.1. The equations of a hypersurface. Let $\bar{M}(c)$ be a complex space form of constant holomorphic sectional curvature $c \neq 0$ and complex dimension $n$. If $c>0$ then $\bar{M}(c)$ is 
a complex projective space $\mathbb{C} P^{n}(c)$ of constant holomorphic sectional curvature $c$. Analogously, if $c<0$ then $\bar{M}(c)$ is a complex hyperbolic space $\mathbb{C} H^{n}(c)$. We denote by $\langle\cdot, \cdot\rangle$ its inner product, by $J$ its Kähler structure, and by $\bar{\nabla}$ its Levi-Civita connection. The curvature tensor is defined by $\bar{R}(X, Y)=\left[\bar{\nabla}_{X}, \bar{\nabla}_{Y}\right]-\bar{\nabla}_{[X, Y]}$, so in this case we have

$$
\bar{R}(X, Y) Z=\frac{c}{4}(\langle Y, Z\rangle X-\langle X, Z\rangle Y+\langle J Y, Z\rangle J X-\langle J X, Z\rangle J Y-2\langle J X, Y\rangle J Z) .
$$

Let $M$ be a connected submanifold of $\bar{M}(c)$. We denote by $\nabla$ and $R$ its Levi-Civita connection and its curvature tensor respectively. By $T M$ and $\nu M$ we denote the tangent and normal bundles of $M$. We use the symbol $\Gamma(\cdot)$ to refer to the smooth sections of any vector bundle. Let $X, Y, Z, W \in \Gamma(T M)$ and $\xi \in \Gamma(\nu M)$.

The second fundamental form $I I$ of $M$ is defined by the Gauss formula as $\bar{\nabla}_{X} Y=\nabla_{X} Y+$ $I I(X, Y)$. The Weingarten formula is then written as $\bar{\nabla}_{X} \xi=-S_{\xi} X+\nabla_{X}^{\perp} \xi$, where $S_{\xi}$ is the shape operator with respect to $\xi$ and $\nabla^{\perp}$ is the induced normal connection on $\nu M$. The second fundamental form and the shape operator are related by $\left\langle S_{\xi} X, Y\right\rangle=\langle I I(X, Y), \xi\rangle$.

Now let $M$ be a connected real hypersurface of $\bar{M}(c)$. The word 'real' emphasizes the fact that the real codimension is one. Fix $\xi \in \Gamma(\nu M)$ a (local) unit normal vector field. We write $S$ instead of $S_{\xi}$. The Gauss formula can be rewritten as

$$
\bar{\nabla}_{X} Y=\nabla_{X} Y+\langle S X, Y\rangle \xi
$$

and hence, the Weingarten formula is $S X=-\bar{\nabla}_{X} \xi$. Moreover, the Gauss and Codazzi equations for a hypersurface are

$$
\begin{aligned}
\langle\bar{R}(X, Y) Z, W\rangle & =\langle R(X, Y) Z, W\rangle-\langle S Y, Z\rangle\langle S X, W\rangle+\langle S X, Z\rangle\langle S Y, W\rangle, \text { and } \\
\langle\bar{R}(X, Y) Z, \xi\rangle & =\left\langle\left(\nabla_{X} S\right) Y-\left(\nabla_{Y} S\right) X, Z\right\rangle .
\end{aligned}
$$

We assume from now on that $M$ has constant principal curvatures, that is, the eigenvalues of the shape operator $S$ are constant. For each principal curvature $\lambda$ of $M$ we denote by $T_{\lambda}$ the distribution on $M$ formed by the principal curvature spaces of $\lambda$ along $M$.

The Codazzi equation implies (see [5, Section 2] for a proof)

Lemma 2.1. (i) Let $p \in M$. If the orthogonal projection of $J \xi_{p}$ onto $T_{\alpha}(p)$ is nonzero, then $T_{\alpha}(p)$ is a real subspace of $T_{p} \bar{M}(c)$, that $i s, J T_{\alpha}(p)$ is orthogonal to $T_{\alpha}(p)$.

(ii) Let $X, Y \in \Gamma\left(T_{\alpha}\right)$ and $Z \in \Gamma\left(T_{\beta}\right)$ with $\alpha \neq \beta$. Then

$$
\left\langle\nabla_{X} Y, Z\right\rangle=\frac{c}{4(\alpha-\beta)}(\langle J Y, Z\rangle\langle X, J \xi\rangle+\langle J X, Y\rangle\langle Z, J \xi\rangle+2\langle J X, Z\rangle\langle Y, J \xi\rangle) .
$$

(iii) Let $X \in \Gamma\left(T_{\alpha}\right), Y \in \Gamma\left(T_{\beta}\right)$ and $Z \in \Gamma\left(T_{\gamma}\right)$. Then

$$
\langle\bar{R}(X, Y) Z, \xi\rangle=(\beta-\gamma)\left\langle\nabla_{X} Y, Z\right\rangle-(\alpha-\gamma)\left\langle\nabla_{Y} X, Z\right\rangle .
$$

The Gauss equation implies (again, see [5, Lemma 4] for a proof) 
Lemma 2.2. Let $X \in \Gamma\left(T_{\alpha}\right)$ and $Y \in \Gamma\left(T_{\beta}\right)$, with $\alpha \neq \beta$, be unit vector fields. Then

$$
\begin{aligned}
0= & (\beta-\alpha)\left(-c-4 \alpha \beta-2 c\langle J X, Y\rangle^{2}+8\left\langle\nabla_{X} Y, \nabla_{Y} X\right\rangle-4\left\langle\nabla_{X} X, \nabla_{Y} Y\right\rangle\right) \\
& -4 c\langle J X, Y\rangle(X\langle Y, J \xi\rangle+Y\langle X, J \xi\rangle) \\
& -c\langle X, J \xi\rangle\left(3 Y\langle J X, Y\rangle+\left\langle\nabla_{Y} X, J Y\right\rangle-2\left\langle\nabla_{X} Y, J Y\right\rangle\right) \\
& -c\langle Y, J \xi\rangle\left(3 X\langle J X, Y\rangle-\left\langle\nabla_{X} Y, J X\right\rangle+2\left\langle\nabla_{Y} X, J X\right\rangle\right) .
\end{aligned}
$$

2.2. Discussion of examples. Part (国) of the Main Theorem states that there are no examples of real hypersurfaces with constant principal curvatures in $\mathbb{C} P^{n}(c)$ whose Hopf vector field has $h=2$ nontrivial projections onto the principal curvature spaces of $M$. Thus, we will focus on describing briefly the examples of part (b) of the Main Theorem. These examples where first constructed in 4 and their geometry was studied in 7 .

The connected simple Lie group $G=S U(1, n)$ acts transitively on $\mathbb{C} H^{n}(c)$. Fix a point $o \in \mathbb{C} H^{n}(c)$ and let $K$ be the isotropy group of $G$ at $o$. The subgroup $K$ of $G$ is isomorphic to $S(U(1) U(n))$. Furthermore, $(G, K)$ is a symmetric pair and $\mathbb{C} H^{n}(c)$ may be identified with the quotient $G / K$. Write $\mathfrak{g}$ for the Lie algebra of $G$ and $\mathfrak{k}$ for the Lie algebra of $K$. Let $\mathfrak{g}=\mathfrak{k} \oplus \mathfrak{p}$ be the Cartan decomposition of $\mathfrak{g}$ with respect to $o \in \mathbb{C} H^{n}(c)$. We choose a maximal abelian subspace $\mathfrak{a}$ of $\mathfrak{p}$; then, $\operatorname{dim} \mathfrak{a}=1$ since $\mathbb{C} H^{n}(c)$ has rank one. Let $\mathfrak{g}=\mathfrak{g}_{-2 \alpha} \oplus \mathfrak{g}_{-\alpha} \oplus \mathfrak{g}_{0} \oplus \mathfrak{g}_{\alpha} \oplus \mathfrak{g}_{2 \alpha}$ be the restricted root space decomposition of $\mathfrak{g}$ with respect to $\mathfrak{a}$ and assume that $\alpha$ is a positive root. Then, $\mathfrak{n}=\mathfrak{g}_{\alpha} \oplus \mathfrak{g}_{2 \alpha}$ is a 2-step nilpotent subalgebra of $\mathfrak{g}$ isomorphic to the $(2 n-1)$-dimensional Heisenberg algebra. Furthermore, $\mathfrak{g}=\mathfrak{k} \oplus \mathfrak{a} \oplus \mathfrak{n}$ is an Iwasawa decomposition of $\mathfrak{g}$. If $A$ and $N$ denote the connected subgroups of $G$ whose Lie algebras are $\mathfrak{a}$ and $\mathfrak{n}$, then $G=K A N$ is an Iwasawa decomposition of $G$. The solvable group $A N$ is simply connected and acts simply transitively on $\mathbb{C} H^{n}(c)$. Thus, we can identify $\mathfrak{a} \oplus \mathfrak{n}$ with $T_{o} \mathbb{C} H^{n}(c)$. The Riemannian metric of $\mathbb{C} H^{n}(c)$ induces a left-invariant metric on $A N$ which makes $A N$ isometric to $\mathbb{C} H^{n}(c)$. Similarly, the complex structure $J$ on $T_{o} \mathbb{C} H^{n}(c)$ induces a complex structure on $\mathfrak{a} \oplus \mathfrak{n}$ which we also denote by $J$. We have $J \mathfrak{a}=\mathfrak{g}_{2 \alpha}$, and $\mathfrak{g}_{\alpha}$ is $J$-invariant. Let $B \in \mathfrak{a}$ be a unit vector and define $Z=J B \in \mathfrak{g}_{2 \alpha}$.

Let $\mathfrak{w}$ be a linear subspace of $\mathfrak{g}_{\alpha}$ such that the orthogonal complement $\mathfrak{w}^{\perp}=\mathfrak{g}_{\alpha} \ominus \mathfrak{w}$ of $\mathfrak{w}$ in $\mathfrak{g}_{\alpha}$ has constant Kähler angle $\varphi \in(0, \pi / 2]$, that is, the angle between $J v$ and $\mathfrak{w}^{\perp}$ is $\varphi$ for all nonzero $v \in \mathfrak{w}^{\perp}$. Then, $\varphi=\pi / 2$ if and only if $\mathfrak{w}^{\perp}$ is real, or equivalently, if and only if $J \mathfrak{w}^{\perp}$ is orthogonal to $\mathfrak{w}^{\perp}$. Let $k$ be the dimension of $\mathfrak{w}^{\perp}$. Then, $\mathfrak{s}=\mathfrak{a} \oplus \mathfrak{w} \oplus \mathfrak{g}_{2 \alpha}$ is a subalgebra of $\mathfrak{a} \oplus \mathfrak{n}$. Let $S$ be the connected simply connected subgroup of $A N$ whose Lie algebra is $\mathfrak{s}$. We define the Berndt-Brück submanifolds as [4] (see [16] for $k=1$ )

$$
W_{\varphi}^{2 n-k}=S \cdot o, \quad \text { and } \quad W^{2 n-k}=W_{\pi / 2}^{2 n-k} .
$$

The Berndt-Brück submanifolds $W_{\varphi}^{2 n-k}$ are homogeneous, have normal bundle of rank $k$ and constant Kähler angle $\varphi \in(0, \pi / 2]$, and their second fundamental form $I I$ is given by the trivial symmetric bilinear extension of $I I(Z, P \xi)=(\sin (\varphi) \sqrt{-c} / 2) \xi$ for all $\xi \in \mathfrak{w}^{\perp}$, where $P \xi$ is the orthogonal projection of $J \xi$ onto $T W_{\varphi}^{2 n-k}$. In particular, the submanifolds $W_{\varphi}^{2 n-k}$ are minimal, and ruled by the totally geodesic complex hyperbolic subspaces determined by their maximal holomorphic distribution. If $\varphi=\pi / 2$ then $P=J$ and the Berndt-Brück submanifolds have totally real normal bundle. Conversely [7, Theorem 1] 
Theorem 2.3. Let $M$ be a $(2 n-k)$-dimensional connected submanifold in $\mathbb{C} H^{n}(c), n \geq 2$, with normal bundle $\nu M$ of constant Kähler angle $\varphi \in(0, \pi / 2]$. Assume that there exists a unit vector field $Z$ tangent to the maximal holomorphic subbundle of $T M$ such that the second fundamental form II of $M$ is given by the trivial symmetric bilinear extension of

$$
I I(Z, P \xi)=\sin (\varphi) \frac{\sqrt{-c}}{2} \xi
$$

for all $\xi \in \Gamma(\nu M)$. Then $M$ is holomorphically congruent to an open part of the ruled minimal submanifold $W_{\varphi}^{2 n-k}$.

In particular, the Berndt-Brück submanifolds $W^{2 n-k}$ are determined by the equation $I I(Z, J \xi)=(\sqrt{-c} / 2) \xi$ and the fact that their normal bundle is totally real. Geometrically, they are constructed in the following way. Fix a horosphere $\mathcal{H}$ in a totally geodesic real hyperbolic space $\mathbb{R} H^{k+1}(c) \subset \mathbb{C} H^{n}(c)$. Attach at each point the totally geodesic $\mathbb{C} H^{n-k}(c)$ which is tangent to the orthogonal complement of the complex span of the tangent space of $\mathcal{H}$ at $p$. The resulting submanifold is congruent to $W^{2 n-k}$.

Let $N_{K}^{0}(S)$ denote the connected component of the identity transformation of the normalizer of $S$ in $K$. Then, $N_{K}^{0}(S) S$ acts on $\mathbb{C} H^{n}(c)$ with cohomogeneity one and $W_{\varphi}^{2 n-k}=$ $N_{K}^{0}(S) S \cdot o$. If $k>1$, then the principal orbits of $N_{K}^{0}(S) S$ are tubes around $W_{\varphi}^{2 n-k}$. If $k=1$, then $\varphi=\pi / 2$, the action of $N_{K}^{0}(S) S$ is orbit equivalent to the action of $S$, and its orbits form a homogeneous foliation on $\mathbb{C} H^{n}(c)$ that was first studied in [3].

Let $M$ be a principal orbit of $N_{K}^{0}(S) S$. If $\varphi \in(0, \pi / 2)$ then the Hopf vector field of $M$ has $h=3$ nontrivial projections onto the principal curvature spaces of $M$. If $\varphi=\pi / 2$, then the Hopf vector field of $M$ has $h=2$ nontrivial projections onto the principal curvature spaces of $M$. The objective of part (B) of the Main Theorem is to give a geometric characterization of the tubes around $W^{2 n-k}, k \in\{2, \ldots, n-1\}$, and the equidistant hypersurfaces to $W^{2 n-1}$.

\section{Proof of the Main Theorem}

In this section we prove the Main Theorem. Our main goal is to describe accurately the eigenvalue structure of a real hypersurface in the conditions of the Main Theorem (Theorem 3.12). Then we finish the proof using standard Jacobi field theory (\$3.6).

3.1. Notation and setup. Let $M$ be a connected real hypersurface with $g>1$ distinct constant principal curvatures in a complex space form $\bar{M}(c)$. Since the calculations that follow are local we may assume that we have a globally defined unit normal vector field $\xi$. We denote by $\lambda_{1}, \ldots, \lambda_{g}$ the principal curvatures of $M$.

By assumption, the number of nontrivial projections of $J \xi$ onto the principal curvature distributions $T_{\lambda_{i}}, i \in\{1, \ldots, g\}$, is $h=2$. By relabeling the indices we may also assume that $J \xi$ has nontrivial projection onto $T_{\lambda_{1}}$ and $T_{\lambda_{2}}$. Hence, there exist unit vectors fields $U_{i} \in \Gamma\left(T_{\lambda_{i}}\right), i \in\{1,2\}$, and positive smooth functions $b_{i}: M \rightarrow \mathbb{R}, i \in\{1,2\}$, such that

$$
J \xi=b_{1} U_{1}+b_{2} U_{2} .
$$

Obviously, $b_{1}^{2}+b_{2}^{2}=1$. Moreover, 
Lemma 3.1. We have $g \geq 3,\left\langle J U_{1}, U_{2}\right\rangle=0$ and there exists a unit vector field $A \in$ $\Gamma\left(\oplus_{k=3}^{g} T_{\lambda_{k}}\right)$ such that

$$
\begin{aligned}
J U_{i} & =(-1)^{i} b_{j} A-b_{i} \xi, \quad(i, j \in\{1,2\}, i \neq j), \\
J A & =b_{2} U_{1}-b_{1} U_{2} .
\end{aligned}
$$

Proof. The proof is similar to that of [5, Lemma 7], so we just sketch it. We will assume in what follows $i, j \in\{1,2\}, i \neq j$, and $k \in\{3, \ldots, g\}$.

Since $T_{\lambda_{i}}, i \in\{1,2\}$, is real by Lemma 2.1 (ii) we can write $J U_{i}=\left\langle J U_{i}, U_{j}\right\rangle U_{j}+W_{i j}+$ $\sum_{k=3}^{g} W_{i k}-b_{i} \xi$, where $W_{i j} \in \Gamma\left(T_{\lambda_{j}} \ominus \mathbb{R} U_{j}\right)$ and $W_{i k} \in \Gamma\left(T_{\lambda_{k}}\right)$. (Here and henceforth, the symbol $\ominus$ is used to denote orthogonal complement.) From $J \xi=b_{1} U_{1}+b_{2} U_{2}$ we get

$$
-\xi=J^{2} \xi=b_{2}\left(\left\langle J U_{2}, U_{1}\right\rangle U_{1}+W_{21}\right)+b_{1}\left(\left\langle J U_{1}, U_{2}\right\rangle U_{2}+W_{12}\right)+\sum_{k=3}^{g}\left(b_{1} W_{1 k}+b_{2} W_{2 k}\right)-\xi .
$$

Thus, $g \geq 3,\left\langle J U_{1}, U_{2}\right\rangle=0, W_{12}=W_{21}=0$, and $b_{1} W_{1 k}+b_{2} W_{2 k}=0$ for all $k$. If we define $A \in \Gamma\left(\oplus_{k=3}^{g} T_{\lambda_{k}}\right)$ by $\sum_{k=3}^{g} W_{i k}=(-1)^{i} b_{j} A$, then the last equality implies $\sum_{k=3}^{g} W_{j k}=$ $(-1)^{j} b_{i} A$ (recall $i, j \in\{1,2\}, i \neq j$ ). This gives the desired expression for $J U_{i}, i \in\{1,2\}$. Finally, from $b_{1}^{2}+b_{2}^{2}=1$ and $-U_{1}=J\left(J U_{1}\right)=-b_{2} J A-b_{1} J \xi=-b_{2} J A-U_{1}+b_{2}^{2} U_{1}-b_{1} b_{2} U_{2}$ we obtain $J A=b_{2} U_{1}-b_{1} U_{2}$.

3.2. The vector field $A$. In view of Lemma 3.1 we may write

$$
A=\sum_{k=3}^{g} A_{k}, \text { with } A_{k} \in \Gamma\left(T_{\lambda_{k}}\right), k \in\{3, \ldots g\} .
$$

The aim of this subsection is to show that all but one $A_{k}$ are zero and hence we can assume for example that $A \in \Gamma\left(T_{\lambda_{3}}\right)$ (Proposition 3.3). The main difficulty here is the fact that $g$ is not known. We start with the following

Lemma 3.2. Let $i, j \in\{1,2\}$ with $i \neq j$. Then we have

$$
\nabla_{U_{i}} U_{i}=\sum_{k=3}^{g}(-1)^{j} \frac{3 c b_{1} b_{2}}{4\left(\lambda_{k}-\lambda_{i}\right)} A_{k}, \quad \nabla_{U_{i}} U_{j}=\sum_{k=3}^{g}(-1)^{j}\left(\lambda_{i}-\frac{3 c b_{i}^{2}}{4\left(\lambda_{k}-\lambda_{i}\right)}\right) A_{k} .
$$

Proof. Again, this is quite similar to [5, Lemma 8]. We assume $i, j \in\{1,2\}, i \neq j$, and $k \in\{3, \ldots, g\}$. Let $W_{i} \in \Gamma\left(T_{\lambda_{i}} \ominus \mathbb{R} U_{i}\right)$ and $W_{k} \in \Gamma\left(T_{\lambda_{k}} \ominus \mathbb{R} A_{k}\right)$.

Since $U_{i}$ has unit length we get $\left\langle\nabla_{U_{i}} U_{i}, U_{i}\right\rangle=0$. Lemma 2.1 (iii) yields $\left\langle\nabla_{U_{i}} U_{i}, U_{j}\right\rangle=$ $\left\langle\nabla_{U_{i}} U_{i}, W_{j}\right\rangle=\left\langle\nabla_{U_{i}} U_{i}, W_{k}\right\rangle=0$, and $\left\langle\nabla_{U_{i}} U_{i}, A_{k}\right\rangle=3(-1)^{j} c b_{1} b_{2} /\left(4\left(\lambda_{k}-\lambda_{i}\right)\right)$. From $\bar{\nabla} J=$ 0 , the Weingarten formula, and Lemma 3.1, we obtain $\left\langle W_{i}, \bar{\nabla}_{U_{i}} J \xi\right\rangle=-\lambda_{i}\left\langle W_{i}, J U_{i}\right\rangle=0$. Hence, using $J \xi=b_{1} U_{1}+b_{2} U_{2}$, and Lemma 2.1 (iii), we get

$$
0=U_{i}\left\langle W_{i}, J \xi\right\rangle=\left\langle\nabla_{U_{i}} W_{i}, J \xi\right\rangle+\left\langle W_{i}, \bar{\nabla}_{U_{i}} J \xi\right\rangle=-b_{i}\left\langle\nabla_{U_{i}} U_{i}, W_{i}\right\rangle .
$$

Since $b_{i} \neq 0$ the expression for $\nabla_{U_{i}} U_{i}$ follows.

As $U_{j}$ has unit length, $\left\langle\nabla_{U_{i}} U_{j}, U_{j}\right\rangle=0$. From Lemma 2.1 (iii) we obtain $\left\langle\nabla_{U_{i}} U_{j}, U_{i}\right\rangle=$ $\left\langle\nabla_{U_{i}} U_{j}, W_{i}\right\rangle=0$. Now, the Weingarten formula and Lemma 3.1 imply $\left\langle W_{j}, \bar{\nabla}_{U_{i}} J \xi\right\rangle=$ 
$-\lambda_{i}\left\langle W_{j}, J U_{i}\right\rangle=0$, and thus, Lemma 2.1 (iii), yields

$$
0=U_{i}\left\langle W_{j}, J \xi\right\rangle=\left\langle\nabla_{U_{i}} W_{j}, J \xi\right\rangle+\left\langle W_{j}, \bar{\nabla}_{U_{i}} J \xi\right\rangle=b_{j}\left\langle\nabla_{U_{i}} W_{j}, U_{j}\right\rangle .
$$

This implies $\left\langle\nabla_{U_{i}} W_{j}, U_{j}\right\rangle=0$. A similar calculation gives $\left\langle\nabla_{U_{i}} W_{k}, U_{j}\right\rangle=0$. Finally, by Lemma 2.1 (iii), and Lemma 3.1 we have

$0=U_{i}\left\langle A_{k}, J \xi\right\rangle=\left\langle\nabla_{U_{i}} A_{k}, J \xi\right\rangle+\left\langle A_{k}, \bar{\nabla}_{U_{i}} J \xi\right\rangle=(-1)^{i} \frac{3 c b_{i}^{2} b_{j}}{4\left(\lambda_{k}-\lambda_{i}\right)}-b_{j}\left\langle\nabla_{U_{i}} U_{j}, A_{k}\right\rangle-(-1)^{i} \lambda_{i} b_{j}$,

from where we get $\left\langle\nabla_{U_{i}} U_{j}, A_{k}\right\rangle$. Altogether this yields the formula for $\nabla_{U_{i}} U_{j}$.

Now we can prove the main result of this section.

Proposition 3.3. $A \in \Gamma\left(T_{\lambda_{k}}\right)$ for some $k \in\{3, \ldots, g\}$.

Proof. On the contrary, assume that there exists a point $p \in M$ and two distinct integers $r, s \in\{3, \ldots, g\}$ such that $\left(A_{r}\right)_{p},\left(A_{s}\right)_{p} \neq 0$. Hence, in a neighborhood of $p$ we have $A_{r}, A_{s} \neq 0$ as well. We will work in that neighborhood from now on.

Applying Lemma 2.1 (iii) to the vector fields $U_{1}, U_{2}$, and $A_{k}, k \in\{r, s\}$, and using Lemma 3.2 we easily get

$$
\frac{3 c\left(\lambda_{2}-\lambda_{k}\right)}{4\left(\lambda_{1}-\lambda_{k}\right)} b_{1}^{2}+\frac{3 c\left(\lambda_{1}-\lambda_{k}\right)}{4\left(\lambda_{2}-\lambda_{k}\right)} b_{2}^{2}=-\frac{c}{4}-\lambda_{1}\left(\lambda_{2}-\lambda_{k}\right)-\lambda_{2}\left(\lambda_{1}-\lambda_{k}\right), \quad k \in\{r, s\} .
$$

Together with $b_{1}^{2}+b_{2}^{2}=1$, this yields a linear system of three equations with unknowns $b_{1}^{2}$ and $b_{2}^{2}$. This system must be compatible. We show it is determined (that is, it has a unique solution). If it were not, the rank of the system would, at most, be one. In particular,

$$
\left|\begin{array}{cc}
\frac{3 c\left(\lambda_{2}-\lambda_{k}\right)}{4\left(\lambda_{1}-\lambda_{k}\right)} & \frac{3 c\left(\lambda_{1}-\lambda_{k}\right)}{4\left(\lambda_{2}-\lambda_{k}\right)} \\
1 & 1
\end{array}\right|=3 c \frac{\left(\lambda_{2}-\lambda_{1}\right)\left(\lambda_{1}+\lambda_{2}-2 \lambda_{k}\right)}{4\left(\lambda_{1}-\lambda_{k}\right)\left(\lambda_{2}-\lambda_{k}\right)}=0, \quad k \in\{r, s\},
$$

which implies $\lambda_{1}+\lambda_{2}-2 \lambda_{k}=0, k \in\{r, s\}$, and hence $\lambda_{r}=\lambda_{s}$, contradiction. We conclude that the above system is determined. Therefore, we can find an expression for $b_{1}^{2}$ and $b_{2}^{2}$ in terms of the principal curvatures and $c$. Since these are constant, it follows that $b_{1}$ and $b_{2}$ are constant.

We take $i, j \in\{1,2\}, i \neq j$, and $k \in\{r, s\}$. Since $b_{i}$ is constant and $U_{i}$ has unit length, using $J \xi=b_{1} U_{1}+b_{2} U_{2}$, the Weingarten formula, and Lemma 3.1 we get

$$
0=A_{k}\left(b_{i}\right)=A_{k}\left\langle U_{i}, J \xi\right\rangle=\left\langle\nabla_{A_{k}} U_{i}, J \xi\right\rangle+\left\langle U_{i}, \bar{\nabla}_{A_{k}} J \xi\right\rangle=b_{j}\left\langle\nabla_{A_{k}} U_{i}, U_{j}\right\rangle-(-1)^{j} b_{j} \lambda_{k},
$$

and thus, $\left\langle\nabla_{A_{k}} U_{i}, U_{j}\right\rangle=(-1)^{j} \lambda_{k}$. Taking this, Lemma 3.1, and Lemma 3.2 into account, Lemma 2.1 (iii) for $A_{k}, U_{1}$ and $U_{2}$ yields

$\frac{c}{4}\left(2 b_{2}^{2}-b_{1}^{2}\right)=\left\langle\bar{R}\left(A_{k}, U_{1}\right) U_{2}, \xi\right\rangle=\left(\lambda_{1}-\lambda_{2}\right) \lambda_{k}+\left(\lambda_{k}-\lambda_{2}\right)\left(\lambda_{1}-\frac{3 c b_{1}^{2}}{4\left(\lambda_{k}-\lambda_{1}\right)}\right), \quad k \in\{r, s\}$.

We can rearrange this as:

$$
\left(\frac{c}{4}-\frac{3 c\left(\lambda_{k}-\lambda_{2}\right)}{4\left(\lambda_{k}-\lambda_{1}\right)}\right) b_{1}^{2}-\frac{c}{2} b_{2}^{2}=\left(\lambda_{2}-\lambda_{1}\right) \lambda_{k}+\lambda_{1}\left(\lambda_{2}-\lambda_{k}\right), \quad k \in\{r, s\} .
$$


Hence, (11), (2), and $b_{1}^{2}+b_{2}^{2}=1$ give a linear system of five equations with unknowns $b_{1}^{2}$ and $b_{2}^{2}$. This system is compatible by assumption, so it has rank two. Then, all minors of order three of the augmented matrix of the system vanish. This implies (take (1), (2), and $b_{1}^{2}+b_{2}^{2}=1$, with $k \in\{r, s\}$, and then both equations in (2) and $\left.b_{1}^{2}+b_{2}^{2}=1\right)$ :

$$
\begin{aligned}
\frac{3 c\left(\lambda_{1}-\lambda_{2}\right)^{2}\left(-12 \lambda_{k}^{2}+8 \lambda_{1} \lambda_{k}+8 \lambda_{2} \lambda_{k}+c-4 \lambda_{1} \lambda_{2}\right)}{16\left(\lambda_{1}-\lambda_{k}\right)\left(\lambda_{k}-\lambda_{2}\right)} & =0, k \in\{r, s\}, \\
\text { (4) } \frac{3 c\left(\lambda_{2}-\lambda_{1}\right)\left(\lambda_{r}-\lambda_{s}\right)\left(4 \lambda_{1}^{2}-4 \lambda_{r} \lambda_{1}-4 \lambda_{s} \lambda_{1}+c+2 \lambda_{2} \lambda_{r}+2 \lambda_{2} \lambda_{s}\right)}{8\left(\lambda_{1}-\lambda_{r}\right)\left(\lambda_{1}-\lambda_{s}\right)} & =0 .
\end{aligned}
$$

In particular, (3) implies $-12 \lambda_{k}^{2}+8 \lambda_{1} \lambda_{k}+8 \lambda_{2} \lambda_{k}+c-4 \lambda_{1} \lambda_{2}=0$. Putting $k=r$ and $k=s$, and subtracting, we get $4\left(2 \lambda_{1}+2 \lambda_{2}-3 \lambda_{r}-3 \lambda_{s}\right)\left(\lambda_{r}-\lambda_{s}\right)=0$, from where we obtain $\lambda_{r}+\lambda_{s}=2\left(\lambda_{1}+\lambda_{2}\right) / 3$. Taking this into account, (41) gives $\left(4 \lambda_{1}^{2}-4 \lambda_{1} \lambda_{2}+4 \lambda_{2}^{2}+3 c\right) / 3=0$. The discriminant of $-12 \lambda_{k}^{2}+8 \lambda_{1} \lambda_{k}+8 \lambda_{2} \lambda_{k}+c-4 \lambda_{1} \lambda_{2}=0$ as a quadratic equation in $\lambda_{k}$ is precisely $16\left(4 \lambda_{1}^{2}-4 \lambda_{1} \lambda_{2}+4 \lambda_{2}^{2}+3 c\right)$, so this discriminant vanishes. As a consequence, this quadratic equation has a unique solution and hence $\lambda_{r}=\lambda_{s}$. This is a contradiction. Therefore, all but one $A_{k}, k \in\{3, \ldots, g\}$, are zero for each $p$. The result follows by continuity.

3.3. Some properties of the principal curvature spaces. In view of Proposition 3.3 , we may assume from now on that $A \in \Gamma\left(T_{\lambda_{3}}\right)$. Moreover, we can choose an orientation on $M$ and a relabeling of the indices so that

$$
\lambda_{1}<\lambda_{2}, \quad \text { and } \quad \lambda_{3} \geq 0 .
$$

We will follow this convention from now on.

First we calculate some covariant derivatives.

Lemma 3.4. Let $i, j \in\{1,2\}$ with $i \neq j$. Then we have

$$
\begin{aligned}
\nabla_{U_{i}} U_{i} & =(-1)^{j} \frac{3 c b_{1} b_{2}}{4\left(\lambda_{3}-\lambda_{i}\right)} A, \\
\nabla_{U_{i}} U_{j} & =(-1)^{j}\left(\lambda_{i}-\frac{3 c b_{i}^{2}}{4\left(\lambda_{3}-\lambda_{i}\right)}\right) A, \\
\nabla_{U_{i}} A & =(-1)^{i} \frac{3 c b_{1} b_{2}}{4\left(\lambda_{3}-\lambda_{i}\right)} U_{i}+(-1)^{i}\left(\lambda_{i}-\frac{3 c b_{i}^{2}}{4\left(\lambda_{3}-\lambda_{i}\right)}\right) U_{j}, \\
\nabla_{A} U_{i} & =\frac{(-1)^{j}}{\lambda_{i}-\lambda_{j}}\left(\frac{c\left(2 b_{j}^{2}-b_{i}^{2}\right)}{4}+\left(\lambda_{j}-\lambda_{3}\right)\left(\lambda_{i}-\frac{3 c b_{i}^{2}}{4\left(\lambda_{3}-\lambda_{i}\right)}\right)\right) U_{j}, \\
\nabla_{A} A & =0 .
\end{aligned}
$$

Proof. The proof is similar to that of [5, Lemma 8]. Equations (5]) and (66) are a direct consequence of Lemma 3.2 and Proposition 3.3. Assume $i, j \in\{1,2\}, i \neq j$, and $k \in$ $\{4, \ldots, g\}$. Let $W_{i} \in \Gamma\left(T_{\lambda_{i}} \ominus \mathbb{R} U_{i}\right), W_{3} \in \Gamma\left(T_{\lambda_{3}} \ominus \mathbb{R} A\right)$ and $W_{k} \in \Gamma\left(T_{\lambda_{k}}\right)$. 
According to (5) and (6), in order to prove (17) we have to show $\left\langle\nabla_{U_{i}} A, A\right\rangle=0$ (obvious because $A$ is a unit vector field), and $\left\langle\nabla_{U_{i}} A, W_{l}\right\rangle=0$ for all $l \in\{1, \ldots, g\}$. The latter follows after using $\bar{\nabla} J=0$, the Weingarten formula, Lemma 3.1, and (15), with

$$
\begin{aligned}
0 & =U_{i}\left\langle J U_{i}, W_{l}\right\rangle=\left\langle\bar{\nabla}_{U_{i}} J U_{i}, W_{l}\right\rangle+\left\langle J U_{i}, \bar{\nabla}_{U_{i}} W_{l}\right\rangle \\
& =-\left\langle\nabla_{U_{i}} U_{i}, J W_{l}\right\rangle+(-1)^{i} b_{j}\left\langle A, \nabla_{U_{i}} W_{l}\right\rangle-b_{i}\left\langle\xi, \bar{\nabla}_{U_{i}} W_{l}\right\rangle=(-1)^{j} b_{j}\left\langle\nabla_{U_{i}} A, W_{l}\right\rangle .
\end{aligned}
$$

We now prove (8). Obviously, $\left\langle\nabla_{A} U_{i}, U_{i}\right\rangle=0$, and $\left\langle\nabla_{A} U_{i}, A\right\rangle=0$ by Lemma 2.1 (ii). Applying Lemma 2.1 (iii) to $A, U_{i}$ and $U_{j}$, using Lemma 3.1 and (6), gives

$$
\frac{c}{4}(-1)^{i}\left(b_{i}^{2}-2 b_{j}^{2}\right)=\left(\lambda_{i}-\lambda_{j}\right)\left\langle\nabla_{A} U_{i}, U_{j}\right\rangle-\left(\lambda_{3}-\lambda_{j}\right)(-1)^{i}\left(\lambda_{i}-\frac{3 c b_{i}^{2}}{4\left(\lambda_{3}-\lambda_{i}\right)}\right),
$$

from where we get $\left\langle\nabla_{A} U_{i}, U_{j}\right\rangle$. For $l \in\{j, 3, \ldots, g\}$, a similar argument with Lemma 2.1 (iii) applied to $A, U_{i}$, and $W_{l}$, taking Lemma 3.1 and (7) into account, yields $\left\langle\nabla_{A} U_{i}, W_{l}\right\rangle=0$. Finally, the previous equality (interchanging $i$ and $j$ and putting $l=i$ ) gives

$$
\begin{aligned}
0 & =A\left\langle W_{i}, J \xi\right\rangle=\left\langle\nabla_{A} W_{i}, J \xi\right\rangle+\left\langle W_{i}, \bar{\nabla}_{A} J \xi\right\rangle \\
& =b_{i}\left\langle\nabla_{A} W_{i}, U_{i}\right\rangle+b_{j}\left\langle\nabla_{A} W_{i}, U_{j}\right\rangle-\lambda_{3}\left\langle W_{i}, J A\right\rangle=-b_{i}\left\langle\nabla_{A} U_{i}, W_{i}\right\rangle .
\end{aligned}
$$

Altogether this proves (8) .

We have $\left\langle\nabla_{A} A, A\right\rangle=0$, and $\left\langle\nabla_{A} A, U_{i}\right\rangle=\left\langle\nabla_{A} A, W_{l}\right\rangle=0$ for all $l \in\{1,2,4, \ldots, g\}$ by Lemma 2.1 (iii). From $\bar{\nabla} J=0$, (8) , Lemma 3.1, and the Weingarten formula we get

$$
\begin{aligned}
0 & =A\left\langle J U_{i}, W_{3}\right\rangle=\left\langle\bar{\nabla}_{A} J U_{i}, W_{3}\right\rangle+\left\langle J U_{i}, \bar{\nabla}_{A} W_{3}\right\rangle \\
& =-\left\langle\nabla_{A} U_{i}, J W_{3}\right\rangle+(-1)^{i} b_{j}\left\langle A, \nabla_{A} W_{3}\right\rangle-b_{i}\left\langle\xi, \bar{\nabla}_{A} W_{3}\right\rangle=(-1)^{j} b_{j}\left\langle\nabla_{A} A, W_{3}\right\rangle .
\end{aligned}
$$

from where (9) follows.

Our main difficulty from now on is the fact that the number $g$ of principal curvatures is not known. In fact, the aim of Subsection 3.4 is to obtain a bound on $g$. An important step in the proof is the following

Proposition 3.5. The functions $b_{1}$ and $b_{2}$ are constant. In fact

$$
b_{i}^{2}=\frac{4\left(\lambda_{j}-2 \lambda_{3}\right)\left(\lambda_{i}-\lambda_{3}\right)^{2}}{c\left(\lambda_{i}-\lambda_{j}\right)}, \quad(i, j \in\{1,2\}, i \neq j) .
$$

Moreover, $c-4 \lambda_{1} \lambda_{2}+8\left(\lambda_{1}+\lambda_{2}\right) \lambda_{3}-12 \lambda_{3}^{2}=0$.

Proof. First we show that the functions $b_{1}$ and $b_{2}$ are constant.

We apply Lemma 2.2 to $U_{1}$ and $U_{2}$, using Lemma 3.1 and Lemma 3.4,

$$
\begin{aligned}
0= & \left(\lambda_{2}-\lambda_{1}\right)\left(-c-4 \lambda_{1} \lambda_{2}+8\left\langle\nabla_{U_{1}} U_{2}, \nabla_{U_{2}} U_{1}\right\rangle-4\left\langle\nabla_{U_{1}} U_{1}, \nabla_{U_{2}} U_{2}\right\rangle\right) \\
& -c b_{1}\left(\left\langle\nabla_{U_{2}} U_{1}, J U_{2}\right\rangle-2\left\langle\nabla_{U_{1}} U_{2}, J U_{2}\right\rangle\right)-c b_{2}\left(-\left\langle\nabla_{U_{1}} U_{2}, J U_{1}\right\rangle+2\left\langle\nabla_{U_{2}} U_{1}, J U_{1}\right\rangle\right) \\
= & -\left(\lambda_{2}-\lambda_{1}\right)\left(c+12 \lambda_{1} \lambda_{2}\right)-\frac{3 c^{2}}{2\left(\lambda_{3}-\lambda_{1}\right)} b_{1}^{4}+\frac{3 c^{2}}{2\left(\lambda_{3}-\lambda_{2}\right)} b_{2}^{4}+\frac{3 c^{2}\left(\lambda_{1}-\lambda_{2}\right)}{\left(\lambda_{3}-\lambda_{1}\right)\left(\lambda_{3}-\lambda_{2}\right)} b_{1}^{2} b_{2}^{2} \\
& +\frac{c\left(6 \lambda_{2}^{2}-7 \lambda_{1} \lambda_{2}-2 \lambda_{1}^{2}+2 \lambda_{1} \lambda_{3}+\lambda_{2} \lambda_{3}\right)}{\lambda_{3}-\lambda_{1}} b_{1}^{2}-\frac{c\left(6 \lambda_{1}^{2}-7 \lambda_{1} \lambda_{2}-2 \lambda_{2}^{2}+2 \lambda_{2} \lambda_{3}+\lambda_{1} \lambda_{3}\right)}{\lambda_{3}-\lambda_{2}} b_{2}^{2} .
\end{aligned}
$$


Now we substitute $b_{2}^{2}$ by $1-b_{1}^{2}$ to get

$$
0=\frac{9 c^{2}\left(\lambda_{2}-\lambda_{1}\right)}{2\left(\lambda_{3}-\lambda_{1}\right)\left(\lambda_{3}-\lambda_{2}\right)} b_{1}^{4}+\Lambda_{1} b_{1}^{2}+\Lambda_{0}
$$

where $\Lambda_{1}$ and $\Lambda_{0}$ are constants depending on $c, \lambda_{1}, \lambda_{2}$, and $\lambda_{3}$. This equation is a quadratic equation in $b_{1}^{2}$ and the coefficient of $b_{1}^{4}$ does not vanish. Hence, it has at most two real solutions depending on the constants $c, \lambda_{1}, \lambda_{2}$ and $\lambda_{3}$. Since $M$ is connected it follows that $b_{1}$ and $b_{2}$ are constant.

From the argument above one might derive an explicit expression for $b_{i}, i \in\{1,2\}$. However, that expression would involve square roots that would make later calculations difficult. Instead, we use the constancy of these functions to give an alternative formula which is easier to handle. For $i \in\{1,2\}$, using lemmas 3.1 and 3.4, and the Weingarten formula, we get

$$
\begin{aligned}
0 & =A\left(b_{i}\right)=A\left\langle U_{i}, J \xi\right\rangle=\left\langle\nabla_{A} U_{i}, J \xi\right\rangle+\left\langle U_{i}, \bar{\nabla}_{A} J \xi\right\rangle=b_{j}\left\langle\nabla_{A} U_{i}, U_{j}\right\rangle-\lambda_{3}\left\langle U_{i}, J A\right\rangle \\
& =(-1)^{i} b_{j}\left(c \frac{-\lambda_{i}+3 \lambda_{j}-2 \lambda_{3}}{4\left(\lambda_{i}-\lambda_{j}\right)\left(\lambda_{3}-\lambda_{i}\right)} b_{i}^{2}-\frac{c}{2\left(\lambda_{i}-\lambda_{j}\right)} b_{j}^{2}+\frac{2 \lambda_{i} \lambda_{3}-\lambda_{j} \lambda_{3}-\lambda_{i} \lambda_{j}}{\lambda_{i}-\lambda_{j}}\right) .
\end{aligned}
$$

Together with $b_{1}^{2}+b_{2}^{2}=1$, this gives a linear system of three equations with unknowns $b_{1}^{2}$ and $b_{2}^{2}$. Since this system is compatible by hypothesis, its rank is two and hence the determinant of its augmented matrix is zero. This implies

$$
\frac{3 c}{16\left(\lambda_{1}-\lambda_{3}\right)\left(\lambda_{2}-\lambda_{3}\right)}\left(c-4 \lambda_{1} \lambda_{2}+8 \lambda_{3}\left(\lambda_{1}+\lambda_{2}\right)-12 \lambda_{3}^{2}\right)=0 .
$$

Solving the above system is only a matter of linear algebra. After some calculations we get $b_{i}^{2}=4\left(\lambda_{j}-2 \lambda_{3}\right)\left(\lambda_{i}-\lambda_{3}\right)^{2} /\left(c\left(\lambda_{i}-\lambda_{j}\right)\right)$ from where the result follows.

We are now able to derive an important relation among $\lambda_{1}, \lambda_{2}$ and $\lambda_{3}$.

Proposition 3.6. We have $c<0$. In this case, we get

$$
\lambda_{i}=\frac{1}{2}\left(3 \lambda_{3}+(-1)^{i} \sqrt{-c-3 \lambda_{3}^{2}}\right), \quad(i, j \in\{1,2\}, i \neq j) .
$$

In particular, $\lambda_{1}<\lambda_{3}<\lambda_{2}$. Moreover, $c+4 \lambda_{3}^{2}<0$, or equivalently, $0 \leq \lambda_{3}<\sqrt{-c} / 2$.

Proof. Let $i, j \in\{1,2\}$ with $i \neq j$. Using Lemma 3.1, the constancy of $b_{i}$, and then Lemma 3.4, we get by Lemma 2.2 applied to $U_{i}$ and $A$

$$
\begin{aligned}
0= & \left(\lambda_{3}-\lambda_{i}\right)\left(-c-4 \lambda_{i} \lambda_{3}-2 c b_{j}^{2}+8\left\langle\nabla_{U_{i}} A, \nabla_{A} U_{i}\right\rangle\right) \\
& -c b_{i}\left((-1)^{i} b_{i}\left\langle\nabla_{A} U_{i}, U_{j}\right\rangle-2(-1)^{j} b_{j}\left\langle\nabla_{U_{i}} A, U_{i}\right\rangle-2(-1)^{i} b_{i}\left\langle\nabla_{U_{i}} A, U_{j}\right\rangle\right) \\
= & \frac{c^{2}\left(\lambda_{i}-15 \lambda_{j}+14 \lambda_{3}\right)}{4\left(\lambda_{3}-\lambda_{i}\right)\left(\lambda_{i}-\lambda_{j}\right)} b_{i}^{4}+\frac{c^{2}\left(-10 \lambda_{i}+3 \lambda_{j}+7 \lambda_{3}\right)}{2\left(\lambda_{3}-\lambda_{i}\right)\left(\lambda_{i}-\lambda_{j}\right)} b_{i}^{2} b_{j}^{2}-\frac{11 c \lambda_{i}\left(\lambda_{3}-\lambda_{j}\right)}{\lambda_{i}-\lambda_{j}} b_{i}^{2} \\
& -\frac{2 c\left(\lambda_{3}-\lambda_{i}\right)\left(3 \lambda_{i}-\lambda_{j}\right)}{\lambda_{i}-\lambda_{j}} b_{j}^{2}-\frac{\left(\lambda_{3}-\lambda_{i}\right)\left(c \lambda_{i}-c \lambda_{j}+8 \lambda_{i}^{2} \lambda_{j}-4 \lambda_{3} \lambda_{i} \lambda_{j}-4 \lambda_{3} \lambda_{i}^{2}\right)}{\lambda_{i}-\lambda_{j}} .
\end{aligned}
$$


Now substituting $b_{i}^{2}, i \in\{1,2\}$, by the expressions given in Proposition 3.5, after multiplying by $\left(\lambda_{j}-\lambda_{i}\right) /\left(\lambda_{i}-\lambda_{3}\right)$ and some long calculations we get

$$
72 \lambda_{3}^{3}-48 \lambda_{i} \lambda_{3}^{2}-108 \lambda_{j} \lambda_{3}^{2}+4 \lambda_{i}^{2} \lambda_{3}+32 \lambda_{j}^{2} \lambda_{3}+72 \lambda_{i} \lambda_{j} \lambda_{3}-16 \lambda_{i} \lambda_{j}^{2}-c \lambda_{i}-8 \lambda_{i}^{2} \lambda_{j}+c \lambda_{j}=0 .
$$

Subtracting the above equation for $i=2$ from the one with $i=1$ we get $2\left(\lambda_{1}-\lambda_{2}\right)(c-$ $\left.4 \lambda_{1} \lambda_{2}+14 \lambda_{3}\left(\lambda_{1}+\lambda_{2}\right)-30 \lambda_{3}^{2}\right)=0$. Combining this with $c-4 \lambda_{1} \lambda_{2}+8\left(\lambda_{1}+\lambda_{2}\right) \lambda_{3}-12 \lambda_{3}^{2}=0$ (Proposition [3.5), we get $6 \lambda_{3}\left(\lambda_{1}+\lambda_{2}-3 \lambda_{3}\right)=0$. If $\lambda_{3}=0$, then the equation above gives $c\left(\lambda_{i}-\lambda_{j}\right)+8 \lambda_{i}^{2} \lambda_{j}+16 \lambda_{i} \lambda_{j}^{2}=0$, which combined with Proposition 3.5 yields $3 c\left(\lambda_{1}+\lambda_{2}\right)=0$. This implies $\lambda_{1}+\lambda_{2}-3 \lambda_{3}=\lambda_{1}+\lambda_{2}=0$, so it suffices to deal with the case $\lambda_{1}+\lambda_{2}-3 \lambda_{3}=0$. In this situation we substitute $\lambda_{1}$ by $-\lambda_{2}+3 \lambda_{3}$ in the equation in Proposition 3.5, thus obtaining $c+4 \lambda_{2}^{2}-12 \lambda_{2} \lambda_{3}+12 \lambda_{3}^{2}=0$. This is a quadratic equation with unknown $\lambda_{2}$ and discriminant $-c-3 \lambda_{3}^{2}$. So that this discriminant is nonnegative we already need $c<0$, proving the first claim of this proposition. The solution to this equation is one of

$$
\frac{1}{2}\left(3 \lambda_{3} \pm \sqrt{-c-3 \lambda_{3}^{2}}\right)
$$

On the other hand, $\lambda_{1}$ is also one of the two values above. Since $\lambda_{1}<\lambda_{2}$ by hypothesis, we get $c+3 \lambda_{3}^{2}<0$ and $\lambda_{i}=\frac{1}{2}\left(3 \lambda_{3}+(-1)^{i} \sqrt{-c-3 \lambda_{3}^{2}}\right)$.

Finally, we show that $0 \leq \lambda_{3}<\sqrt{-c} / 2$. We already know that $0 \leq \lambda_{3}<\sqrt{-c / 3}$. Substituting the above expression for $\lambda_{i}, i \in\{1,2\}$, in Proposition 3.5 we get

$$
b_{i}^{2}=-\frac{\left((-1)^{i} \lambda_{3}+\sqrt{-c-3 \lambda_{3}^{2}}\right)^{3}}{2 c \sqrt{-c-3 \lambda_{3}^{2}}}, \quad i \in\{1,2\} .
$$

If $\sqrt{-c} / 2 \leq \lambda_{3}<\sqrt{-c / 3}$, then $-c-4 \lambda_{3}^{2} \leq 0$, and hence $-\lambda_{3}+\sqrt{-c-3 \lambda_{3}^{2}} \leq 0$. This implies $b_{1}^{2} \leq 0$, a contradiction. Therefore $0 \leq \lambda_{3}<\sqrt{-c} / 2$ and the result follows.

Proposition 3.6 already implies that there are no hypersurfaces with constant principal curvatures in $\mathbb{C} P^{n}(c), n \geq 2$, whose Hopf vector field has $h=2$ nontrivial projections onto the principal curvature spaces. From now on we can assume $c<0$.

Corollary 3.7. The distribution $T_{\lambda_{k}}$ is totally real for all $k \in\{4, \ldots, g\}$.

Proof. Let $k \in\{4, \ldots, g\}$ and take unit vector fields $V_{k}, W_{k} \in \Gamma\left(T_{\lambda_{k}}\right)$. Using the Weingarten equation, Lemma 2.1 (iii), Proposition 3.5, and $\lambda_{1}+\lambda_{2}-3 \lambda_{3}=0$ (by Proposition 3.6) we get

$$
\begin{aligned}
0 & =V_{k}\left\langle W_{k}, J \xi\right\rangle=\left\langle\nabla_{V_{k}} W_{k}, b_{1} U_{1}+b_{2} U_{2}\right\rangle+\left\langle W_{k}, \bar{\nabla}_{V_{k}} J \xi\right\rangle \\
& =\left(\frac{c b_{1}^{2}}{4\left(\lambda_{k}-\lambda_{1}\right)}+\frac{c b_{2}^{2}}{4\left(\lambda_{k}-\lambda_{2}\right)}-\lambda_{k}\right)\left\langle J V_{k}, W_{k}\right\rangle=\frac{\left(\lambda_{3}-\lambda_{k}\right)^{3}}{\left(\lambda_{k}-\lambda_{1}\right)\left(\lambda_{k}-\lambda_{2}\right)}\left\langle J V_{k}, W_{k}\right\rangle
\end{aligned}
$$

Since $\lambda_{k} \neq \lambda_{3}$, we get $\left\langle J V_{k}, W_{k}\right\rangle=0$. As $V_{k}$ and $W_{k}$ are arbitrary, the result follows. 
3.4. A bound on the number of principal curvatures. In this section we show, using the Gauss equation and some inequalities involving the principal curvatures, that the number $g$ of distinct principal curvatures satisfies $g \in\{3,4\}$. This allows us to obtain further properties of the principal curvature spaces (see Proposition 3.11). We start with the Gauss equation.

Lemma 3.8. Let us denote by $(\cdot)_{i}, i \in\{1,2\}$, the orthogonal projection onto the distribution $T_{\lambda_{i}} \ominus \mathbb{R} U_{i}$, and by $(\cdot)_{k}, k \in\{4, \ldots, g\}$, the orthogonal projection onto $T_{\lambda_{k}} \cdot B y\|\cdot\|$ we denote the norm of a vector. Then we have:

(i) Let $i \in\{1,2\}$ and $W_{i} \in \Gamma\left(T_{\lambda_{i}} \ominus \mathbb{R} U_{i}\right)$ be a unit vector field. If $j \in\{1,2\}$ and $j \neq i$ then

$$
0=-\left(c+4 \lambda_{3} \lambda_{i}\right)+8 \frac{\lambda_{i}-\lambda_{j}}{\lambda_{3}-\lambda_{j}}\left\|\left(\nabla_{A} W_{i}\right)_{j}\right\|^{2}+8 \sum_{k=4}^{g} \frac{\lambda_{i}-\lambda_{k}}{\lambda_{3}-\lambda_{k}}\left\|\left(\nabla_{A} W_{i}\right)_{k}\right\|^{2} .
$$

(ii) Let $k \in\{4, \ldots, g\}$ and $W_{k} \in \Gamma\left(T_{\lambda_{k}}\right)$ be a unit vector field. Then

$$
\begin{aligned}
0= & -\left(c+4 \lambda_{3} \lambda_{k}\right)+8 \frac{\lambda_{k}-\lambda_{1}}{\lambda_{3}-\lambda_{1}}\left\|\left(\nabla_{A} W_{k}\right)_{1}\right\|^{2}+8 \frac{\lambda_{k}-\lambda_{2}}{\lambda_{3}-\lambda_{2}}\left\|\left(\nabla_{A} W_{k}\right)_{2}\right\|^{2} \\
& +8 \sum_{l=4, l \neq k}^{g} \frac{\lambda_{k}-\lambda_{l}}{\lambda_{3}-\lambda_{l}}\left\|\left(\nabla_{A} W_{k}\right)_{l}\right\|^{2} .
\end{aligned}
$$

Proof. As usual, let $i, j \in\{1,2\}$ with $i \neq j$, and $k \in\{4, \ldots, g\}$.

Let $W_{i} \in \Gamma\left(T_{\lambda_{i}}\right) \ominus \mathbb{R} U_{i}$ be a unit vector field. Applying Lemma 2.2 to $W_{i}$ and $A$ we get

$$
-c-4 \lambda_{3} \lambda_{i}+8\left\langle\nabla_{W_{i}} A, \nabla_{A} W_{i}\right\rangle=0 .
$$

If $W_{3} \in \Gamma\left(T_{\lambda_{3}}\right)$, we get from Lemma 2.1 (iii) that $\left\langle\nabla_{A} W_{i}, W_{3}\right\rangle=0$. This and Lemma 3.4 yield $\nabla_{A} W_{i} \in \Gamma\left(\left(T_{\lambda_{1}} \ominus \mathbb{R} U_{1}\right) \oplus\left(T_{\lambda_{2}} \ominus \mathbb{R} U_{2}\right) \oplus T_{\lambda_{4}} \oplus \cdots \oplus T_{\lambda_{g}}\right)$. Similarly, Lemma 2.1 (iii) implies $\nabla_{W_{i}} A \in \Gamma\left(T_{\lambda_{j}} \oplus\left(T_{\lambda_{3}} \ominus \mathbb{R} A\right) \oplus T_{\lambda_{4}} \oplus \cdots \oplus T_{\lambda_{q}}\right)$. Hence $\left\langle\nabla_{W_{i}} A, \nabla_{A} W_{i}\right\rangle=\left\langle\nabla_{W_{i}} A,\left(\nabla_{A} W_{i}\right)_{j}\right\rangle+$ $\sum_{k=4}^{g}\left\langle\nabla_{W_{i}} A,\left(\nabla_{A} W_{i}\right)_{k}\right\rangle$. For each addend of this sum we apply Lemma 2.1 (iii). Since $\left\langle\bar{R}\left(W_{i}, A\right)\left(\nabla_{A} W_{i}\right)_{l}, \xi\right\rangle=0$ for all $l \in\{j, 4, \ldots, g\}$ we get

$$
\left\langle\nabla_{W_{i}} A, \nabla_{A} W_{i}\right\rangle=\frac{\lambda_{i}-\lambda_{j}}{\lambda_{3}-\lambda_{j}}\left\langle\nabla_{A} W_{i},\left(\nabla_{A} W_{i}\right)_{j}\right\rangle+\sum_{k=4}^{g} \frac{\lambda_{i}-\lambda_{k}}{\lambda_{3}-\lambda_{k}}\left\langle\nabla_{A} W_{i},\left(\nabla_{A} W_{i}\right)_{k}\right\rangle .
$$

Now, part (ii) follows by substituting the previous expression in (10).

Part (ii) follows in a similar way by applying Lemma 2.2 to $W_{k}$ and $A$.

We will use the following technical lemma several times in what follows.

Lemma 3.9. Assume $g \geq 4$ and let $k \in\{4, \ldots, g\}$. Assume that one of the following statements is true:

(i) $\operatorname{dim} T_{\lambda_{1}}=\operatorname{dim} T_{\lambda_{2}}=1$, or

(ii) $\operatorname{dim} T_{\lambda_{1}}=1$ and $\lambda_{k}<\lambda_{2}$, or

(iii) $\lambda_{1}<\lambda_{k}<\lambda_{2}$.

Then, $c+4 \lambda_{3} \lambda_{k} \geq 0$. 
Proof. On the contrary, assume $c+4 \lambda_{3} \lambda_{k}<0$. Let $W_{k} \in \Gamma\left(T_{\lambda_{k}}\right)$ be a (local) unit vector field. When we apply Lemma 3.8 (iii) to $W_{k}$, any of the assumptions ensures that the first three addends of the equation given in Lemma 3.8 (iii) are nonnegative with the first one strictly positive. This already implies $g>4$. In this case, it follows that there exists $r \in\{4, \ldots, g\}, r \neq k$, such that $\left(\lambda_{k}-\lambda_{r}\right) /\left(\lambda_{3}-\lambda_{r}\right)<0$. We may choose $\lambda_{r}$ to be the principal curvature that minimizes $\left|\lambda_{3}-\lambda_{l}\right|$ among all $\lambda_{l}, l \in\{4, \ldots, g\}, l \neq k$, with $\left(\lambda_{k}-\lambda_{l}\right) /\left(\lambda_{3}-\lambda_{l}\right)<0$. In particular we have

$$
\text { either } \lambda_{k}<\lambda_{r}<\lambda_{3} \text { or } \lambda_{3}<\lambda_{r}<\lambda_{k} \text {. }
$$

It follows that $\lambda_{r}$ satisfies the same assumption as $\lambda_{k}$ : this is obvious for (ii) and a consequence of (11) and $\lambda_{1}<\lambda_{3}<\lambda_{2}$ for (iii) and (iii). Using (11), $\lambda_{3} \geq 0, c+4 \lambda_{3}^{2}<0$ (Proposition 3.6), and $c+4 \lambda_{3} \lambda_{k}<0$, we also get $c+4 \lambda_{3} \lambda_{r} \leq c+4 \lambda_{3} \max \left\{\lambda_{3}, \lambda_{k}\right\}<0$. Thus we may apply Lemma 3.8 (iii) to a unit vector field $W_{r} \in \Gamma\left(T_{\lambda_{r}}\right)$, from where it follows, as before, that there exists $s \in\{4, \ldots, g\}, s \neq r$, such that $\left(\lambda_{r}-\lambda_{s}\right) /\left(\lambda_{3}-\lambda_{s}\right)<0$. This implies either $\lambda_{r}<\lambda_{s}<\lambda_{3}$ or $\lambda_{3}<\lambda_{s}<\lambda_{r}$, and taking (11) into account we easily obtain

$$
\text { either } \quad \lambda_{k}<\lambda_{r}<\lambda_{s}<\lambda_{3} \text { or } \quad \lambda_{3}<\lambda_{s}<\lambda_{r}<\lambda_{k} \text {. }
$$

In both cases (12) yields $s \neq k,\left(\lambda_{k}-\lambda_{s}\right) /\left(\lambda_{3}-\lambda_{s}\right)<0$, and $\left|\lambda_{3}-\lambda_{s}\right|<\left|\lambda_{3}-\lambda_{r}\right|$. This contradicts the definition of $\lambda_{r}$. Therefore, $c+4 \lambda_{3} \lambda_{k} \geq 0$.

From the previous lemma we easily derive the first important consequence.

Proposition 3.10. We have $\operatorname{dim} T_{\lambda_{1}}=1$.

Proof. On the contrary, assume $\operatorname{dim} T_{\lambda_{1}}>1$ and let $W_{1} \in \Gamma\left(T_{\lambda_{1}} \ominus \mathbb{R} U_{1}\right)$ be a (local) unit vector field. Since $c+4 \lambda_{1} \lambda_{3} \leq c+4 \lambda_{3}^{2}<0$ by Proposition [3.6. from Lemma [3.8 (ii) we deduce the existence of $k \in\{4, \ldots, g\}$ such that $\left(\lambda_{1}-\lambda_{k}\right) /\left(\lambda_{3}-\lambda_{k}\right)<0$. Since $\lambda_{1}<\lambda_{3}$ we get $\lambda_{1}<\lambda_{k}<\lambda_{3}<\lambda_{2}$ and hence Lemma 3.9 (iii) yields $c+4 \lambda_{3} \lambda_{k} \geq 0$. This contradicts $c+4 \lambda_{3} \lambda_{k} \leq c+4 \lambda_{3}^{2}<0$. Therefore $\operatorname{dim} T_{\lambda_{1}}=1$.

This is the most crucial step of the proof.

Proposition 3.11. We have

(i) $g \in\{3,4\}$.

(ii) If $g=3$ and $\operatorname{dim} T_{\lambda_{2}}>1$ then $\lambda_{1}=0, \lambda_{2}=\frac{\sqrt{-3 c}}{2}$, and $\lambda_{3}=\frac{\sqrt{-c}}{2 \sqrt{3}}$.

(iii) If $g=4$ then $\operatorname{dim} T_{\lambda_{2}}=1,0 \neq \lambda_{3} \neq \frac{\sqrt{-c}}{2 \sqrt{3}}$, and $\lambda_{4}=-\frac{c}{4 \lambda_{3}}$.

Proof. If $g=3$ and $\operatorname{dim} T_{\lambda_{2}}>1$, take a (local) unit $W_{2} \in \Gamma\left(T_{\lambda_{2}} \ominus \mathbb{R} U_{2}\right)$ and apply Lemma 3.8 (ii). Note that the last two addends vanish since $\operatorname{dim} T_{\lambda_{1}}=1$ and $g=3$. Then, $c+4 \lambda_{2} \lambda_{3}=0$, and from Proposition 3.6 we get $\lambda_{1}=0, \lambda_{2}=\sqrt{-3 c} / 2$, and $\lambda_{3}=\sqrt{-c} /(2 \sqrt{3})$. This implies (iii).

Assume $g \geq 4$. We first have $\lambda_{3}<\lambda_{k}$ for all $k \in\{4, \ldots, g\}$; otherwise, if $\lambda_{k}<\lambda_{3}<\lambda_{2}$ we would get $c+4 \lambda_{3} \lambda_{k} \leq c+4 \lambda_{3}^{2}<0$ contradicting Lemma 3.9 (iii) (by Proposition 3.10).

We show that $\operatorname{dim} T_{\lambda_{2}}=1$. On the contrary, assume $\operatorname{dim} T_{\lambda_{2}}>1$ and let $W_{2} \in \Gamma\left(T_{\lambda_{2}} \ominus\right.$ $\mathbb{R} U_{2}$ ) be a (local) unit vector field. If $c+4 \lambda_{2} \lambda_{3}<0$, then Lemma 3.8 (ii) applied to $W_{2}$ 
(and taking Proposition 3.10 into account) implies that there exists $k \in\{4, \ldots, g\}$ such that $\left(\lambda_{2}-\lambda_{k}\right) /\left(\lambda_{3}-\lambda_{k}\right)<0$. Then, $\lambda_{3}<\lambda_{k}<\lambda_{2}$, and thus $c+4 \lambda_{3} \lambda_{k} \leq c+4 \lambda_{3} \lambda_{2}<0$, which contradicts Lemma 3.9 (iii). Hence we can assume from now on that $c+4 \lambda_{2} \lambda_{3} \geq 0$. This inequality does not hold if $\lambda_{3}=0$ so we already get $\lambda_{3}>0$.

We claim that there exists $r \in\{4, \ldots, g\}$ such that $\lambda_{2}<\lambda_{r}$. If $c+4 \lambda_{2} \lambda_{3}=0$, then the assertion is true for all $k \geq 4$; otherwise, if $\lambda_{k}<\lambda_{2}$, we would get $c+4 \lambda_{3} \lambda_{k}<c+4 \lambda_{3} \lambda_{2}=0$, contradicting Lemma 3.9 (ii). Hence, we have to prove our claim for the case $c+4 \lambda_{2} \lambda_{3}>0$. In this case we apply Lemma 3.8 (1i) to $W_{2}$. Then, there exists $r \in\{4, \ldots, g\}$ such that $\left(\lambda_{2}-\lambda_{r}\right) /\left(\lambda_{3}-\lambda_{r}\right)>0$. Since $\lambda_{3}<\lambda_{r}$ this implies $\lambda_{2}<\lambda_{r}$ as claimed.

In any case, there exists $r \in\{4, \ldots, g\}$ such that $\lambda_{2}<\lambda_{r}$. In fact, we may assume that $\lambda_{r}$ is the largest principal curvature. Now, we have $c+4 \lambda_{3} \lambda_{r}>c+4 \lambda_{3} \lambda_{2} \geq 0$, and hence Lemma 3.8 (iii) applied to a unit vector field $W_{r} \in \Gamma\left(T_{\lambda_{r}}\right)$ implies the existence of $l \in\{4, \ldots, g\}, l \neq r$, such that $\left(\lambda_{r}-\lambda_{l}\right) /\left(\lambda_{3}-\lambda_{l}\right)>0$. Since $\lambda_{3}<\lambda_{l}$, we get $\lambda_{r}<\lambda_{l}$ which contradicts the fact that $\lambda_{r}$ is the largest principal curvature. Altogether this implies $\operatorname{dim} T_{\lambda_{2}}=1$.

From Lemma 3.9 (ii) we obtain $c+4 \lambda_{3} \lambda_{k} \geq 0$ for all $k \geq 4$. In particular this implies $\lambda_{3}>0$. Assume that for some $r \in\{4, \ldots, g\}$ we have strict inequality $c+4 \lambda_{3} \lambda_{r}>0$ and let $\lambda_{r}, r \in\{4, \ldots, g\}$, be the largest principal curvature satisfying this condition. Applying Lemma 3.8 (iil) once more to a unit $W_{r} \in \Gamma\left(T_{\lambda_{r}}\right)$ (note that the second addend now vanishes) yields the existence of $l \in\{4, \ldots, g\}, l \neq r$, such that $\left(\lambda_{r}-\lambda_{l}\right) /\left(\lambda_{3}-\lambda_{l}\right)>0$. Since $\lambda_{3}<\lambda_{l}$ we get $\lambda_{r}<\lambda_{l}$. Obviously, $c+4 \lambda_{3} \lambda_{l}>c+4 \lambda_{3} \lambda_{r}>0$, which contradicts the fact that $\lambda_{r}$ is the largest principal curvature satisfying this condition.

As a consequence, $c+4 \lambda_{3} \lambda_{k}=0$ for all $k \geq 4$. Since $\lambda_{3} \neq 0$ and the principal curvatures are different, this immediately implies $g=4$ and $\lambda_{4}=-c /\left(4 \lambda_{3}\right)$. Eventually, this also yields $c+4 \lambda_{3} \lambda_{2} \neq 0$ and thus, by Proposition 3.6, $\lambda_{3} \neq \sqrt{-c} /(2 \sqrt{3})$ (otherwise the principal curvatures would not be different). This concludes the proof of (ii) and (iii).

Part (ii) of Proposition 3.11 had already been obtained in [5] by different methods. We have included a proof here as it is almost effortless to do so.

3.5. The eigenvalue structure of the shape operator. We summarize the results obtained so far:

Theorem 3.12. We have:

(a) There are no real hypersurfaces with constant principal curvatures in $\mathbb{C} P^{n}(c), n \geq 2$, whose Hopf vector field has $h=2$ nontrivial projections onto the principal curvature spaces.

(b) Let $M$ be a connected real hypersurface with $g$ distinct constant principal curvatures $\lambda_{1}, \ldots, \lambda_{g}$ in $\mathbb{C} H^{n}(c), n \geq 2$, such that the number of nontrivial projections of its Hopf vector field $J \xi$ onto the principal curvature spaces of $M$ is $h=2$. Then, $g \in\{3,4\}$ and, with a suitable labeling of the principal curvatures and a suitable choice of the normal vector field $\xi$, we have: 
(i) The Hopf vector field can be written as $J \xi=b_{1} U_{1}+b_{2} U_{2}$, where $U_{i} \in \Gamma\left(T_{\lambda_{i}}\right)$, $i \in\{1,2\}$, are unit vector fields, and $b_{1}$ and $b_{2}$ are positive constants satisfying

$$
b_{i}^{2}=\frac{4\left(\lambda_{j}-2 \lambda_{3}\right)\left(\lambda_{i}-\lambda_{3}\right)^{2}}{c\left(\lambda_{i}-\lambda_{j}\right)}, \quad(i, j \in\{1,2\}, i \neq j) .
$$

(ii) There exists a unit vector field $A \in \Gamma\left(T_{\lambda_{3}}\right)$ such that

$$
J U_{i}=(-1)^{i} b_{j} A-b_{i} \xi, \quad(i, j \in\{1,2\}, i \neq j), \quad \text { and } \quad J A=b_{2} U_{1}-b_{1} U_{1} .
$$

(iii) We have $0 \leq \lambda_{3}<\frac{1}{2} \sqrt{-c}$, and

$$
\lambda_{i}=\frac{1}{2}\left(3 \lambda_{3}+(-1)^{i} \sqrt{-c-3 \lambda_{3}^{2}}\right), \quad(i, j \in\{1,2\}, i \neq j) .
$$

(iv) $\operatorname{dim} T_{\lambda_{1}}=1$.

(v) If $g=4$ then $\operatorname{dim} T_{\lambda_{2}}=1$. We define $k=\operatorname{dim} T_{\lambda_{4}}+1$, and thus, $k \in\{2, \ldots, n-1\}$. The distribution $T_{\lambda_{4}}$ is totally real with $J T_{\lambda_{4}} \subset T_{\lambda_{3}} \ominus \mathbb{R} A$,

$$
0 \neq \lambda_{3} \neq \frac{\sqrt{-c}}{2 \sqrt{3}}, \quad \text { and } \quad \lambda_{4}=-\frac{c}{4 \lambda_{3}} .
$$

(vi) If $g=3$ there are two possibilities:

(A) $\operatorname{dim} T_{\lambda_{2}}=1$; in this case we define $k=1$.

(B) $\operatorname{dim} T_{\lambda_{2}}>1$; in this case we define $k=\operatorname{dim} T_{\lambda_{2}} \in\{2, \ldots, n-1\}$ and we have that $T_{\lambda_{2}} \ominus \mathbb{R} U_{2}$ is a real distribution with $J\left(T_{\lambda_{2}} \ominus \mathbb{R} U_{2}\right) \subset T_{\lambda_{3}} \ominus \mathbb{R} A$, and

$$
\lambda_{1}=0, \quad \lambda_{2}=\frac{\sqrt{-3 c}}{2}, \quad \lambda_{3}=\frac{\sqrt{-c}}{2 \sqrt{3}} .
$$

Remark 3.13. Part (国) of Theorem 3.12 already provides a proof for part (国) of the Main Theorem.

We know that $\mathbb{R} U_{1} \oplus \mathbb{R} U_{2} \oplus \mathbb{R} A \oplus \mathbb{R} \xi$ is a complex subbundle on $M$ by Lemma 3.1. Thus, in part (bv) of Theorem 3.12, the fact that $T_{\lambda_{4}}$ is real (Corollary 3.7) implies $J T_{\lambda_{4}} \subset T_{\lambda_{3}} \ominus \mathbb{R} A$ as claimed. Similarly, in Theorem $3.12 \mathrm{~b}(\mathrm{vi}) \mathrm{B}$, the assertion $J\left(T_{\lambda_{2}} \ominus \mathbb{R} U_{2}\right) \subset T_{\lambda_{3}} \ominus \mathbb{R} A$ follows from the fact that $T_{\lambda_{2}}$ is real by Lemma 2.1 (ii).

The definition of $k$ above might seem a bit artificial at the moment, but it will be useful in the next section where we conclude the proof of the Main Theorem $(k-1$ will be the dimension of the kernel of the differential of the map $\left.\Phi^{r}: M \rightarrow \mathbb{C} H^{n}(c), p \mapsto \exp _{p}\left(r \xi_{p}\right)\right)$.

If we examine the proof of our theorem, so far we have actually shown that for any point $p \in M$ there exists a neighborhood of $p$ where the conclusion of Theorem 3.12 is satisfied. However, by the connectedness of $M$ and a continuity argument, it can be easily shown that $M$ is orientable and that the conclusion of Theorem 3.12 is satisfied globally.

3.6. Jacobi field theory and rigidity of focal submanifolds. In this last section we finish the proof of part (b) of the Main Theorem. Since we use standard Jacobi field theory, we provide the reader just with the fundamental details and skip the long calculations. According to [5] we just have to take care of the case $g=4$. However, it is not much 
overload to deal with the two cases simultaneously, so for the sake of completeness we will do so in what follows.

Let $M$ be a real hypersurface of $\mathbb{C} H^{n}(c)$ in the conditions of Theorem 3.12 (b) . For $r \in \mathbb{R}$ we define the map $\Phi^{r}: M \rightarrow \mathbb{C} H^{n}(c), p \mapsto \exp _{p}\left(r \xi_{p}\right)$, where $\exp _{p}$ is the Riemannian exponential map of $\mathbb{C} H^{n}(c)$ at $p$. Then, $\Phi^{r}(M)$ is obtained by moving $M$ a distance $r$ along its normal direction. The singularities of $\Phi^{r}$ are the focal points of $M$. We will find a particular distance $r$ for which $\Phi_{*}^{r}$ has constant rank, where $\Phi_{*}^{r}$ denotes the differential of $\Phi^{r}$. Then we will apply Theorem 2.3 to $\Phi^{r}(M)$ for this choice of $r$. This way, $\Phi^{r}(M)$ will be an open part of the ruled minimal Berndt-Brück submanifold $W^{2 n-k}, k \in\{1, \ldots, n-1\}$, and hence $M$ will be an open part of a tube around this ruled minimal submanifold $W^{2 n-k}$. (If $k=1$ then $M$ will be an equidistant hypersurface to the ruled minimal hypersurface $W^{2 n-1}$ at distance $r$.)

Let $p \in M$ and denote by $\gamma_{p}$ the geodesic determined by the initial conditions $\gamma_{p}(0)=p$ and $\dot{\gamma}_{p}(0)=\xi_{p}$. For any $v \in T_{p} M$ let $B_{v}$ be the parallel vector field along the geodesic $\gamma_{p}$ such that $B_{v}(0)=v$, and let $\zeta_{v}$ be the Jacobi field along $\gamma_{p}$ with initial conditions $\zeta_{v}(0)=v$ and $\zeta^{\prime}(0)=-S_{p} v$. Here ' denotes covariant derivative along $\gamma_{p}$. Since $\zeta_{v}$ is a solution to the differential equation $4 \zeta_{v}^{\prime \prime}-c \zeta_{v}-3 c\left\langle\zeta_{v}, J \dot{\gamma}_{p}\right\rangle J \dot{\gamma}_{p}=0$, if $v \in T_{\lambda_{i}}(p)$ then

$$
\zeta_{v}(t)=f_{i}(t) B_{v}(t)+\langle v, J \xi\rangle g_{i}(t) J \dot{\gamma}_{p}(t),
$$

where

$$
\begin{aligned}
& f_{i}(t)=\cosh \left(\frac{t \sqrt{-c}}{2}\right)-\frac{2 \lambda_{i}}{\sqrt{-c}} \sinh \left(\frac{t \sqrt{-c}}{2}\right) \\
& g_{i}(t)=\left(\cosh \left(\frac{t \sqrt{-c}}{2}\right)-1\right)\left(1+2 \cosh \left(\frac{t \sqrt{-c}}{2}\right)-\frac{2 \lambda_{i}}{\sqrt{-c}} \sinh \left(\frac{t \sqrt{-c}}{2}\right)\right) .
\end{aligned}
$$

We also define the smooth vector field $\eta^{r}$ along $\Phi^{r}$ by $\eta_{p}^{r}=\dot{\gamma}_{p}(r)$. It is known that $\zeta_{v}(r)=\Phi_{*}^{r} v$ and $\zeta_{v}^{\prime}(r)=\bar{\nabla}_{\Phi_{*}^{r} v} \eta^{r}$.

We now determine the value of $r$. Since $0 \leq \lambda_{3}<\sqrt{-c} / 2$ we can find a real number $r \geq 0$ such that

$$
\lambda_{3}=\frac{\sqrt{-c}}{2} \tanh \left(\frac{r \sqrt{-c}}{2}\right) .
$$

Let $p \in M$. We define $u_{i}=\left(U_{i}\right)_{p}, i \in\{1,2\}$. Let $v_{2} \in T_{\lambda_{2}}(p) \ominus \mathbb{R} u_{2}$ and $v_{k} \in T_{\lambda_{k}}(p)$ for $3 \leq k \leq g$ (whenever these spaces are nontrivial). The explicit solution to the Jacobi equation above implies

$$
\begin{gathered}
\left(\Phi_{*}^{r} u_{1}, \Phi_{*}^{r} u_{2}\right)=\left(B_{u_{1}}(r), B_{u_{2}}(r)\right) D(r), \\
\Phi_{*}^{r} v_{2}=0, \quad \Phi_{*}^{r} v_{3}=\operatorname{sech}\left(\frac{t \sqrt{-c}}{2}\right) B_{v_{3}}(r), \quad \Phi_{*}^{r} v_{4}=0,
\end{gathered}
$$

where

$$
D(t)=\left(\begin{array}{cc}
f_{1}(t)+b_{1}^{2} g_{1}(t) & b_{1} b_{2} g_{2}(t) \\
b_{1} b_{2} g_{1}(t) & f_{2}(t)+b_{2}^{2} g_{2}(t)
\end{array}\right) .
$$


Since $\operatorname{det}(D(r))=\operatorname{sech}^{3}(r \sqrt{-c} / 2)$ we conclude that $\Phi_{*}^{r}$ has constant rank $2 n-k$ (see Theorem 3.12 (bv)-(bvi) for the definition of $k$ ). Then, for each point $p \in M$ there exists an open neighborhood $\mathcal{V}$ of $p$ such that $\mathcal{W}=\Phi^{r}(\mathcal{V})$ is an embedded submanifold of $\mathbb{C} H^{n}(c)$ and $\Phi^{r}: \mathcal{V} \rightarrow \mathcal{W}$ is a submersion. (If $k=1$, then $\Phi^{r}$ is actually a local diffeomorphism.)

Let $q=\Phi^{r}(p) \in \mathcal{W}$. The expression above for $\Phi_{*}^{r}$ shows that the tangent space $T_{q} \mathcal{W}$ of $\mathcal{W}$ at $q$ is obtained by parallel translation of $\mathbb{R} u_{1} \oplus \mathbb{R} u_{2} \oplus T_{\lambda_{3}}(p)$ along the geodesic $\gamma_{p}$ from $p=\gamma_{p}(0)$ to $q=\gamma_{p}(r)$. Therefore, the normal space $\nu_{q} \mathcal{W}$ of $\mathcal{W}$ at $q$ is obtained by parallel translation of $\left(\operatorname{ker} \Phi_{* p}^{r}\right) \oplus \mathbb{R} \xi_{p}$ along $\gamma_{p}$ from $p=\gamma_{p}(0)$ to $q=\gamma_{p}(r)$. The latter is $\left(T_{\lambda_{2}} \ominus \mathbb{R} u_{2}\right) \oplus \mathbb{R} \xi_{p}$ if $g=3$ (see Theorem 3.12 (bvil)), or $T_{\lambda_{4}}(p) \oplus \mathbb{R} \xi_{p}$ if $g=4$ (see Theorem $3.12(\mathrm{bv})$ ). In any case, by Theorem 3.12 (bv)-(bvil) it follows that $\mathcal{W}$ has totally real normal bundle of rank $k$.

We have that $\eta_{p}^{r}=B_{\xi_{p}}(r)$ is a unit normal vector of $\mathcal{W}$ at $q$. If $S^{r}$ denotes the shape operator of $\mathcal{W}$, then it is known that $S_{\eta_{p}^{r}}^{r} \Phi_{*}^{r} v=-\left(\zeta_{v}^{\prime}(r)\right)^{\top}$, where $(\cdot)^{\top}$ denotes orthogonal projection onto the tangent space of $\mathcal{W}$. Using the explicit expression for $\zeta_{v}$ above, we get

$$
\left(S_{\eta_{p}^{r}}^{r} B_{u_{1}}(r), S_{\eta_{p}^{r}}^{r} B_{u_{2}}(r)\right)=\left(B_{u_{1}}(r), B_{u_{2}}(r)\right) C(r), \quad \text { and } \quad S_{\eta_{p}^{r}}^{r} B_{v_{3}}(r)=0 \text { for all } v_{3} \in T_{\lambda_{3}}(p),
$$

where $C(r)=-D^{\prime}(r) D(r)^{-1}$. A lengthy and tedious calculation shows that

$$
C(r)=\frac{\sqrt{-c}}{2}\left(\begin{array}{cc}
-2 b_{1} b_{2} & b_{1}^{2}-b_{2}^{2} \\
b_{1}^{2}-b_{2}^{2} & 2 b_{1} b_{2}
\end{array}\right) .
$$

Since $J \eta_{p}^{r}=B_{J \xi_{p}}(r)=b_{1} B_{u_{1}}(r)+b_{2} B_{u_{2}}(r)$, and $B_{J A_{p}}(r)=b_{2} B_{u_{1}}(r)-b_{1} B_{u_{2}}(r)$, the above expression for $C(r)$ implies

$$
S_{\eta_{p}^{r}}^{r} B_{J A_{p}}(r)=-\frac{\sqrt{-c}}{2} J \eta_{p}^{r}, \quad S_{\eta_{p}^{r}}^{r} J \eta_{p}^{r}=-\frac{\sqrt{-c}}{2} B_{J A_{p}}(r),
$$

and $S_{\eta_{p}^{r}}^{r}$ vanishes on the orthogonal complement of $\mathbb{R} J \eta_{p}^{r} \oplus \mathbb{R} B_{J A_{p}}(r)$ in $T_{q} \mathcal{W}$.

We have that $J\left(\nu_{q} \mathcal{W} \ominus \mathbb{R} \eta_{p}^{r}\right)$ is contained in the parallel translation along $\gamma_{p}$ of $T_{\lambda_{3}}(p)$. This follows from Theorem $3.12(\mathrm{bv})$-(bvi) and the fact that $\nu_{q} \mathcal{W} \ominus \mathbb{R} \eta_{p}^{r}$ is the parallel translation along $\gamma_{p}$ from $\gamma_{p}(0)=p$ to $\gamma_{p}(r)=q$ of $T_{\lambda_{2}}(p) \ominus \mathbb{R} u_{2}$ if $g=3$, and of $T_{\lambda_{4}}(p)$ if $g=4$. The linearity of $S_{\eta_{r}^{p}}^{r}$ implies

$$
S_{\eta_{p}^{r}}^{r} J \tilde{\eta}=-\frac{\sqrt{-c}}{2}\left\langle\eta_{p}^{r}, \tilde{\eta}\right\rangle B_{J A_{p}}(r), \text { for all } \tilde{\eta} \in \nu_{q} \mathcal{W} .
$$

It follows from the Gauss formula and $\bar{\nabla} J=0$ that $S_{\tilde{\eta}}^{r} J \eta_{p}^{r}=S_{\eta_{p}^{r}}^{r} J \tilde{\eta}$, and hence, $S_{\tilde{\eta}}^{r} J \eta_{p}^{r}=0$ for all $\tilde{\eta} \in \nu_{q} \mathcal{W} \ominus \mathbb{R} \eta_{p}^{r}$. Let $\alpha$ be a curve in $\left(\Phi^{r}\right)^{-1}(\{q\}) \cap \mathcal{V}$ with $\alpha(0)=p$. Since $\eta_{p}^{r}$ and $\eta_{\alpha(t)}^{r}-\left\langle\eta_{\alpha(t)}^{r}, \eta_{p}^{r}\right\rangle \eta_{p}^{r}$ are perpendicular, $S_{\tilde{\eta}}^{r} J \eta_{p}^{r}=0$, and the linearity of $\eta \mapsto S_{\eta}^{r}$ imply

$$
0=S_{\eta_{\alpha(t)}^{r}-\left\langle\eta_{\alpha(t)}^{r}, \eta_{p}^{r}\right\rangle \eta_{p}^{r}} J \eta_{p}^{r}=S_{\eta_{\alpha(t)}^{r}}^{r} J \eta_{p}^{r}+\frac{\sqrt{-c}}{2}\left\langle\eta_{\alpha(t)}^{r}, \eta_{p}^{r}\right\rangle B_{J A_{p}}(r)
$$

which together with (13) (with $\alpha(t)$ instead of $p$ ) yields

$$
-\frac{\sqrt{-c}}{2}\left\langle\eta_{\alpha(t)}^{r}, \eta_{p}^{r}\right\rangle B_{J A_{p}}(r)=S_{\eta_{\alpha(t)}^{r}}^{r} J \eta_{p}^{r}=-\frac{\sqrt{-c}}{2}\left\langle\eta_{\alpha(t)}^{r}, \eta_{p}^{r}\right\rangle B_{J A_{\alpha(t)}}(r) .
$$


Since $\alpha$ is arbitrary we get that the map $\tilde{p} \mapsto B_{J A_{\tilde{p}}}(r)$ is constant in the connected component $\mathcal{V}_{0}$ of $\left(\Phi^{r}\right)^{-1}(\{q\}) \cap \mathcal{V}$ containing $p$. Thus it makes sense to define the unit vector $z=-B_{J A_{\tilde{p}}}(r) \in T_{q} \mathcal{W}$ for any $\tilde{p} \in \mathcal{V}_{0}$.

We may consider $\eta^{r}$ as a map from $\mathcal{V}_{0}$ to the unit sphere of $\nu_{q} \mathcal{W}$. The tangent space of $\mathcal{V}_{0}$ at $p$ is given by the kernel of $\Phi_{* p}^{r}$. If $v \in \operatorname{ker} \Phi_{* p}^{r}$, then $\eta_{* p}^{r} v=\zeta_{v}^{\prime}(r)$. If $g=3$, then $v \in \operatorname{ker} \Phi_{* p}^{r}=T_{\lambda_{2}}(p) \ominus \mathbb{R} u_{2}$, and $\eta_{* p}^{r}=-\sqrt{-c / 2} B_{v}(r)$. If $g=4$, then $v \in \operatorname{ker} \Phi_{* p}^{r}=T_{\lambda_{4}}(p)$, and $\eta_{* p}^{r} v=-\operatorname{csch}(r \sqrt{-c} / 2) B_{v}(r)$. In any case, we get that $\eta^{r}$ is a local diffeomorphism from $\mathcal{V}_{0}$ into the unit sphere of $\nu_{q} \mathcal{W}$ (note that this is trivial if $g=3$ and $k=1$ ). Hence, $\eta^{r}\left(\mathcal{V}_{0}\right)$ is an open subset of the unit sphere of $\nu_{q} \mathcal{W}$. But since $\eta \mapsto S_{\eta}^{r}$ depends analytically on $\eta$ we conclude

$$
S_{\eta}^{r} J \eta=\frac{\sqrt{-c}}{2} z, \quad S_{\eta}^{r} z=\frac{\sqrt{-c}}{2} J \eta, \quad S_{\eta}^{r} v=0,
$$

for all unit $\eta \in \nu_{q} \mathcal{W}$, and $v \in T_{q} \mathcal{W} \ominus(\mathbb{R} J \eta \oplus \mathbb{R} z)$. Therefore, the second fundamental form $I I^{r}$ of $\mathcal{W}$ at $q$ is given by the trivial symmetric bilinear extension of $I I^{r}(z, J \eta)=(\sqrt{-c} / 2) \eta$ for all $\eta \in \nu_{q} \mathcal{W}$. By construction, $z$ depends smoothly on the point $q \in \mathcal{W}$ and hence gives rise to a vector field $Z$ which is tangent to the maximal holomorphic distribution of $\mathcal{W}$. The relation $S_{\eta}^{r} J \eta=(\sqrt{-c} / 2) Z$ ensures that $Z$ can actually be defined on $\Phi^{r}(M)$, and hence, the second fundamental form of $\Phi^{r}(M)$ is given by the trivial symmetric bilinear extension of $I I^{r}(Z, J \eta)=(\sqrt{-c} / 2) \eta$ for all $\eta \in \Gamma\left(\nu \Phi^{r}(M)\right)$. Since $\Phi^{r}(M)$ has totally real normal bundle of rank $k$ we conclude from Theorem 2.3 , and the remark that follows, that $\Phi^{r}(M)$ is holomorphically congruent to an open part of the ruled minimal Berndt-Brück submanifold $W^{2 n-k}$. This readily implies that $M$ is an open part of a tube (an equidistant hypersurface if $g=3$ and $k=1$ ) of radius $r$ around the ruled minimal Berndt-Brück submanifold $W^{2 n-k}$.

Finally, let us point out that if $g=3$ and $\lambda_{3}=0$, then $r=0$ and $M$ is an open part of the ruled minimal hypersurface $W^{2 n-1}$. Also, if $g=3$ and $k>1$ then $\lambda_{3}=\sqrt{-c} /(2 \sqrt{3})$ according to Theorem $3.12 \mathrm{~b}$ (vi)B, and hence $r=(1 / \sqrt{-c}) \log (2+\sqrt{3})$. The tube around the ruled minimal submanifold $W^{2 n-k}, k>1$, of radius $r=(1 / \sqrt{-c}) \log (2+\sqrt{3})$ has $g=3$

principal curvatures whereas if $r \neq(1 / \sqrt{-c}) \log (2+\sqrt{3})$ the tube of radius $r$ around the ruled minimal submanifold $W^{2 n-k}, k>1$, has $g=4$ principal curvatures.

This finishes the proof of the Main Theorem.

\section{REFERENCES}

[1] U. Abresch: Isoparametric hypersurfaces with four or six distinct principal curvatures. Necessary conditions on the multiplicities, Math. Ann. 264 (1983), 283-302.

[2] J. Berndt: Real hypersurfaces with constant principal curvatures in complex hyperbolic space, $J$. Reine Angew. Math. 395 (1989), 132-141.

[3] J. Berndt: Homogeneous hypersurfaces in hyperbolic spaces, Math. Z. 229 (1998), 589-600.

[4] J. Berndt, M. Brück: Cohomogeneity one actions on hyperbolic spaces, J. Reine Angew. Math. 541 (2001), 209-235.

[5] J. Berndt, J.C. Díaz-Ramos: Real hypersurfaces with constant principal curvatures in complex hyperbolic spaces, J. London Math. Soc. 74 (2006), 778-798. 
[6] J. Berndt, J.C. Díaz-Ramos: Real hypersurfaces with constant principal curvatures in the complex hyperbolic plane, Proc. Amer. Math. Soc., 135 (2007), 3349-3357.

[7] J. Berndt, J. C. Díaz-Ramos: Homogeneous hypersurfaces in complex hyperbolic spaces, Geom. Dedicata, 138 (2009) 129-150.

[8] J. Berndt, H. Tamaru: Cohomogeneity one actions on noncompact symmetric spaces of rank one, Trans. Amer. Math. Soc. 359 (2007), 3425-3438.

[9] É. Cartan: Familles de surfaces isoparamétriques dans les espaces à courbure constante, Ann. Mat. Pura Appl., IV. Ser. 17 (1938), 177-191.

[10] T. Cecil, Q.-S. Chi, G. Jensen: Isoparametric hypersurfaces with four principal curvatures, Ann. of Math. (2) 166 (2007), 1-76.

[11] J. Dorfmeister, E. Neher: Isoparametric hypersurfaces, case $g=6, m=1$, Commun. Algebra 13 (1985), 2299-2368.

[12] W.-Y. Hsiang, H.B. Lawson Jr.: Minimal submanifolds of low cohomogeneity, J. Differential Geom. 5 (1971), 1-38.

[13] D. Ferus, H. Karcher, H.-F. Münzner: Cliffordalgebren und neue isoparametrische Hyperflächen, Math. Z. 177 (1981), 479-502.

[14] S. Immervoll: On the classification of isoparametric hypersurfaces with four principal curvatures in spheres, Ann. of Math. (2) 168 (2008), 1011-1024.

[15] M. Kimura: Real hypersurfaces and complex submanifolds in complex projective space, Trans. Amer. Math. Soc. 296 (1986), 137-149.

[16] M. Lohnherr, H. Reckziegel: On ruled real hypersurfaces in complex space forms, Geom. Dedicata $\mathbf{7 4}$ (1999), 267-286.

[17] S. Montiel: Real hypersurfaces of a complex hyperbolic space, J. Math. Soc. Japan 37 (1985), 515-535.

[18] H. F. Münzner: Isoparametrische Hyperflächen in Sphären, Math. Ann. 251 (1980), 57-71.

[19] R. Niebergall, P. J. Ryan: Real Hypersurfaces in Complex Space Forms, Tight and Taut Submanifolds, MSRI Publications, Volume 32, 1997.

[20] B. Segre: Famiglie di ipersuperficie isoparametriche negli spazi euclidei ad un qualunque numero di dimensioni, Atti Accad. Naz. Lincei Rend. Cl. Sci. Fis. Mat. Natur. (6) 27 (1938), 203-207.

[21] R. Takagi: On homogeneous real hypersurfaces in a complex projective space, Osaka J. Math. 10 (1973), 495-506.

[22] R. Takagi: Real hypersurfaces in a complex projective space with constant principal curvatures, $J$. Math. Soc. Japan 27 (1975), 43-53.

[23] R. Takagi: Real hypersurfaces in a complex projective space with constant principal curvatures, II, J. Math. Soc. Japan 27 (1975), 507-516.

[24] G. Thorbergsson: A survey on isoparametric hypersurfaces and their generalizations, Handbook of differential geometry, Vol. I, 963-995 (North-Holland, Amsterdam, 2000).

[25] Q. M. Wang: Real hypersurfaces with constant principal curvatures in complex projective spaces I, Sci. Sinica Ser. A 26 (1983), 1017-1024.

Department of Geometry and Topology, University of Santiago de Compostela, Spain. E-mail address: josecarlos.diaz@usc.es

Department of Geometry and Topology, University of Santiago de Compostela, Spain. E-mail address: miguel.dominguez@usc.es 